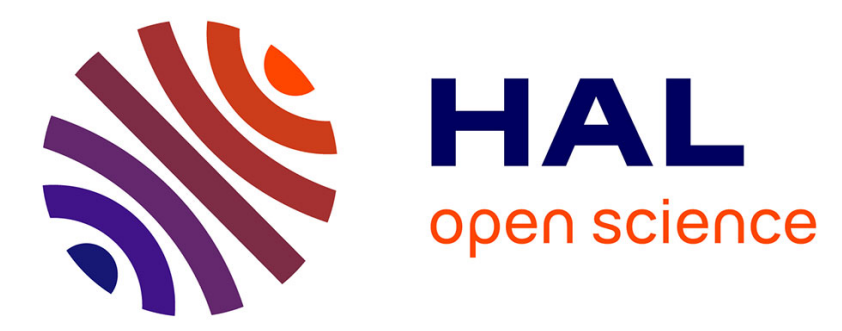

\title{
Vibronic structure of the cyanobutadiyne cation. II. Theoretical exploration of the complex energy landscape of $\mathrm{HC} 5 \mathrm{~N}+$
}

Bérenger Gans, Séverine Boyé-Péronne, Jacques Liévin

\section{- To cite this version:}

Bérenger Gans, Séverine Boyé-Péronne, Jacques Liévin. Vibronic structure of the cyanobutadiyne cation. II. Theoretical exploration of the complex energy landscape of HC $5 \mathrm{~N}+$. Journal of Chemical Physics, 2019, 150 (24), pp.244303. 10.1063/1.5097691 . hal-02167651

\section{HAL Id: hal-02167651 \\ https://hal.science/hal-02167651}

Submitted on 28 Jun 2019

HAL is a multi-disciplinary open access archive for the deposit and dissemination of scientific research documents, whether they are published or not. The documents may come from teaching and research institutions in France or abroad, or from public or private research centers.
L'archive ouverte pluridisciplinaire HAL, est destinée au dépôt et à la diffusion de documents scientifiques de niveau recherche, publiés ou non, émanant des établissements d'enseignement et de recherche français ou étrangers, des laboratoires publics ou privés. 
Bérenger Gans, ${ }^{1}$ Séverine Boyé-Péronne, ${ }^{1}$ and Jacques Liévin ${ }^{2,}$ a)

1) Institut des Sciences Moléculaires d'Orsay (ISMO), CNRS UMR 8214, Univ. Paris-Sud, Université Paris-Saclay, F-91405 Orsay, France

${ }^{2)}$ Service de Chimie Quantique et Photophysique, Université Libre de Bruxelles, CP 160/09, B-1050 Bruxelles, Belgium

(Dated: 17 May 2019)

The results of an extensive $a b$ initio study of the cyanobutadiyne cation, initially motivated by Threshold-Photoelectron Spectroscopy) experiments (see Gans et al., JCP XX XX (2019)), are reported in the present paper. Calculations at the InternallyContracted Multireference Configuration interaction level of theory have been performed to derive the rovibronic properties of the seven lowest electronic states of $\mathrm{HC}_{5} \mathrm{~N}^{+}$. Equilibrium geometries, rotational constants, vibrational frequencies, electric dipole moments and spin-orbit constants have been calculated and compared with experimental data when available. Adiabatic and vertical ionization energies from the neutral ground state, as well as transition energies within the cation electronic manifold are predicted, using the convergence to the Complete Basis Set limit. The accurate description of the complex energy landscape up to $32000 \mathrm{~cm}^{-1}$ above the ionization potential allows to perform Franck-Condon simulations of the photoionization spectrum to the $\mathrm{X}^{+2} \Pi, \mathrm{A}^{+2} \Pi, \mathrm{B}^{+2} \Sigma^{+}$, and $\mathrm{C}^{+2} \Pi$ states, and allows to simulate the $\mathrm{A}^{+2} \Pi \rightarrow \mathrm{X}^{+2} \Pi$ emission spectrum. The vibronic perturbations occurring on the excited potential energy surfaces are revealed and discussed, in particular for the 3 ${ }^{2} \Pi$ surface which presents a double-well topography.

\footnotetext{
a) corresponding author; jlievin@ulb.ac.be
} 


\section{Publishi.hg INTRODUCTION}

The present paper is the second one of a series dealing with the vibronic structure of the cyanobutadiyne cation $\left(\mathrm{HC}_{5} \mathrm{~N}^{+}\right)$up to several $\mathrm{eV}$ above the cationic ground state. The first paper $^{1}$ (hereafter referred to as Paper I) was focused on the Threshold PhotoElectron Spectrum (TPES) of $\mathrm{HC}_{5} \mathrm{~N}$ investigated by synchrotron-based experiments. It has been recorded on the DESIRS beamline of the SOLEIL synchrotron facility and covers the 84 $000-120000 \mathrm{~cm}^{-1}$ range with a $120 \mathrm{~cm}^{-1}$ spectral resolution. The assignment of the vibronic structures observed in the TPES has been achieved with the support of the highlevel $a b$ initio calculations detailed in the present paper. These calculations turned out to be challenging mainly because of the multiconfigurational features charaterizing such $\pi$ conjugated systems. Let us recall indeed the linear structure of the parent neutral system $\mathrm{HC}_{5} \mathrm{~N}$, which exhibits an alternation of single and triple bonds

$$
\mathrm{H}-\mathrm{C}_{1} \equiv \mathrm{C}_{2}-\mathrm{C}_{3} \equiv \mathrm{C}_{4}-\mathrm{C}_{5} \equiv \mathrm{N} \text {. }
$$

The reorganization of this $\pi$ structure upon ionization depends on the concerned cationic state, introducing important multiconfigurational effects, as shown by the analysis of the wavefunctions. Another computational challenge comes from the complex energy landscape of cyanobutadiyne cation resulting from numerous potential energy surface crossings. The experimental TPES exhibits four vibrationally resolved electronic states of the cation $\left(\mathrm{X}^{+2} \Pi, \mathrm{A}^{+2} \Pi, \mathrm{B}^{+2} \Sigma^{+}\right.$, and $\left.\mathrm{C}^{+2} \Pi\right)$ very close in energy with other electronic states predicted by the $a b$ initio calculations but unobservable through single-photon ionization within the electric dipolar approximation. The density of states gives rise to several state crossings, which complicate the topography of the potential energy surfaces (PES) in some regions of the coordinate space. In addition, as $\mathrm{HC}_{5} \mathrm{~N}^{+}$is linear, the degenerate electronic states are also affected by the Renner-Teller effect.

So far, only few theoretical papers have investigated the electronic and vibrational structures of the $\mathrm{HC}_{5} \mathrm{~N}^{+}$cation. In 1996, Lee ${ }^{2}$ used unrestricted BLYP theory with 6-311G* and $6-311 \mathrm{G}^{* *}$ basis sets to compute the geometry, vibrational frequencies, and dipole moments of the ground state of several cyano- and dicyanopolyacetylene cations, including cyanobutadiyne. Tatchen and Marian $^{3}$ performed spin-orbit calculations for the $\mathrm{X}^{+2} \Pi$ electronic ground state using an effective one-electron mean-field spin-orbit hamiltonian. 
Publishifigter on, Lee and Adamowicz ${ }^{4}$ calculated the vertical transition energy at the CASSCF and CASPT2 levels of theory for the lowest $\pi \rightarrow \pi^{\star}$ electronic excitation, corresponding to the $\mathrm{A}^{+2} \Pi \leftarrow \mathrm{X}^{+2} \Pi$ transition. Cao and Peyerimhoff ${ }^{5}$ extended this work to the higher doublet excited electronic states by performing multireference configuration interaction (MRCI) calculations to predict the vertical electronic transition energies and oscillator strengths of transitions from the $\mathrm{X}^{+2} \Pi$ cationic ground state towards the seven lowest excited states. The goal of the study was to provide reliable information on the electronic absorption spectrum of several linear isoelectronic species such as $\mathrm{HC}_{5} \mathrm{~N}^{+}$cation for which measurements had been recorded by Maier's group ${ }^{6}$ in rare gas matrix experiments. No vibrational frequencies were determined for the excited electronic states

In Paper I, only the outlines of the calculations were described and the presented $a b$ initio results were restricted to adiabatic photoionization threshold calculations for the four lowest electronic states, and to vibrational structure calculations of interest for the TPES. The theoretical study is more complete and detailed here. It extends to the seven low-lying states and investigates more properties, which may be helpful for further spectroscopic studies of the cation. This includes the characterization of the spin-orbit coupling, the Renner-Teller effect and the electronic excitation properties (energies and transition electric dipole moments). It also discusses the topography of the PES and includes a Franck-Condon simulation of the absorption spectrum of the cation. All these results are presented and discussed in the present paper, where they are also compared with previous theoretical studies when available (comparison with experimental results was done in Paper I).

The paper is organized as follows. We present the method of calculations in Section II. The results are developed and discussed in Section III. They include the calculated equilibrium properties (geometries, vibrational frequencies, dipole moments and spin-orbit constants), ionization and transition energies. Finally, the investigation of the topography of the crossing potential energy surfaces is discussed. 


\section{METHODS OF CALCULATION}

\section{A. Electronic problem}

All calculations were performed with the MOLPRO program package ${ }^{7,8}$ running on the HYDRA and VEGA clusters of the ULB/VUB computer center in Brussels (Belgium).

As pointed out in our previous works on the cyano- and dicyanoacetylene cations, ${ }^{9-12}$ such linear $\pi$-bonded systems require high-level multireference $a b$ initio methods to describe their excited electronic structure. We have adopted for this purpose the accurate Internally Contracted MultiReference Configuration Interaction (ic-MRCI) method, ${ }^{13,14}$ using molecular orbitals (MOs) which are optimized for the multireference wavefunction by a State Averaged Complete Active Space Self-Consistent Field (SA-CASSCF) calculation. ${ }^{15,16}$ The state averaging has been used systematically for the calculation of excited states of a given symmetry. As MOLPRO only works with abelian group symmetries, we have also applied the state averaging to ensure the exact two-fold degeneracy of the potential energy surfaces of $\Lambda \neq 0$ electronic states, using the symmetry of an abelian subgroup of $\mathrm{C}_{\infty \mathrm{v}}\left(\mathrm{C}_{2 \mathrm{v}}\right.$ and $\mathrm{C}_{\mathrm{s}}$ for linear and bent geometries, respectively).

The complete active space (CAS) has been defined based on test-calculations, which showed that the multiconfigurational character of the states of interest essentially resulted from the mixing between the four low-lying $\pi$ orbitals, in agreement with our observations on similar systems. ${ }^{9-12}$ We thus adopted a $\mathrm{CAS}(13,9)$ in all calculations, which means that according to $\mathrm{C}_{2 \mathrm{v}}$ labelling, 13 electrons are distributed into 9 active MOs (one $\mathrm{a}_{1}$, four $\mathrm{b}_{1}$, and four $\mathrm{b}_{2}$, correlating to one $\sigma$ and four $\pi$ in $\mathrm{C}_{\infty \mathrm{v}}$ symmetry), in addition to a core of twelve doubly-occupied $\mathrm{a}_{1}(\sigma)$ MOs. These active MOs are drawn in Figure 1, by order of decreasing stability from $13 \sigma$ to $4 \pi$. The lone pair of the $\mathrm{N}$ atom is clearly represented by the $130 \mathrm{MO}$, while the four $\pi$ MOs describe the delocalization of the $\pi$ structure along the nuclear chain, with a combination of bonding and antibonding features between adjacent atoms. One clearly sees that the bonding characters of the $\mathrm{C}-\mathrm{C}, \mathrm{C} \equiv \mathrm{C}$, and $\mathrm{C} \equiv \mathrm{N}$ bonds are well represented by this set of MOs. Antibonding contributions are also included, with an increasing number of nodal planes (0 to 3 ) from $1 \pi$ to $4 \pi$, respectively. Higher energy valence MOs ( $14 \sigma$ to $19 \sigma$ and $5 \pi$ to $6 \pi$ ) bring additional antibonding contributions, but according to our test-calculations, they do not participate to the multiconfigurational 


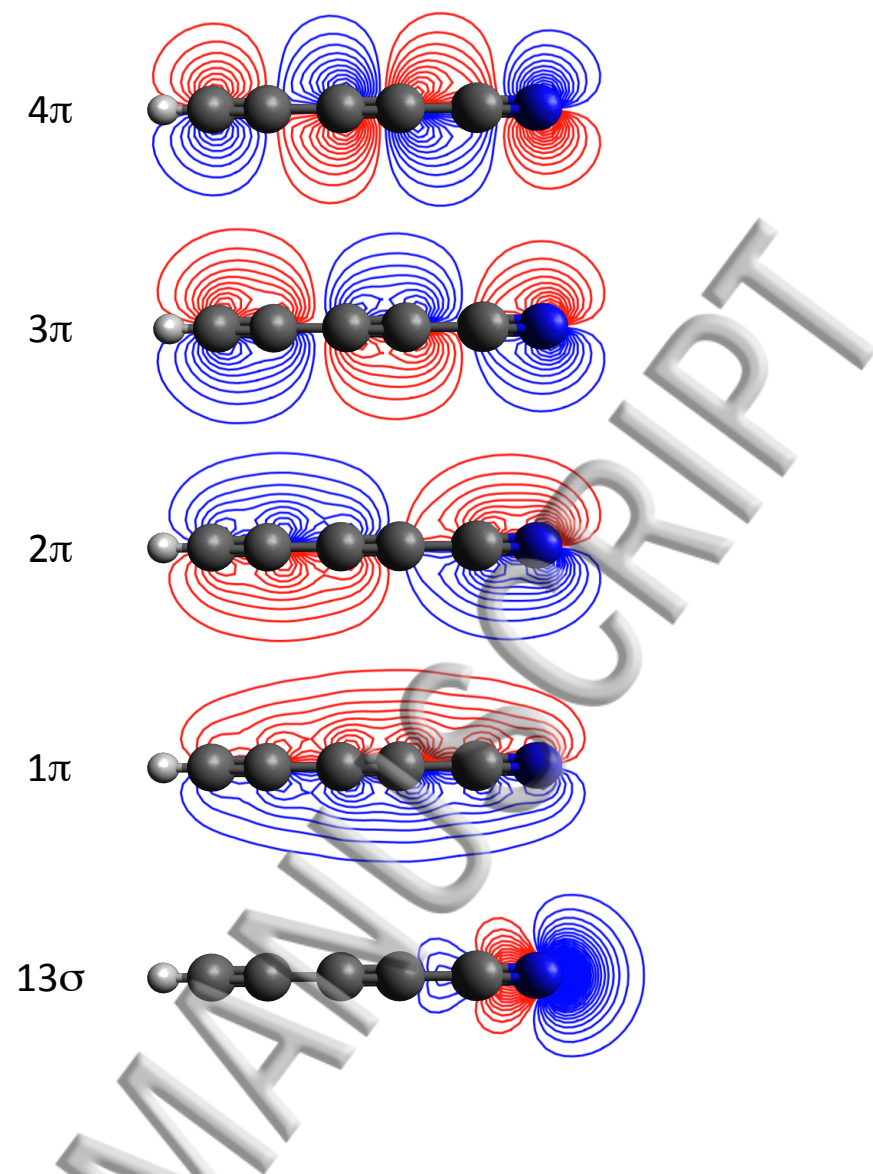

FIG. 1. Isocontour profiles of the CASSCF valence molecular orbitals of $\mathrm{HC}_{5} \mathrm{~N}^{+}$(isocontour step values of 0.02 ).

mixing and have been excluded from the CAS.

This CAS has been used in all SA-CASSCF calculations, but also in the building step of the multireference wavefunctions at ic-MRCI level. However the core of doubly excited MOs has been reduced from 12 to $6 \mathrm{MOs}$ in order to include all valence electrons in the single and double excitation process. The ic-MRCI wavefunctions are expanded over 10 to 90 millions of contracted configuration state functions depending on the state and the basis set. The efficient MRCIc code, ${ }^{17}$ allowing the contraction of the single excitations in addition to the double ones, has been used for all calculations performed on the lower state of a given symmetry.

All ic-MRCI energies were corrected for the contributions of unlinked clusters by means of the Davidson correction ${ }^{18}$ adapted to a relaxed reference, ${ }^{19}$ leading to a level of calculation denoted below as ic-MRCI+Q. 
Publishing]Dunning's correlation consistent cc-pVnZ and aug-cc-pVnZ basis set series (VnZ and AVnZ for short, respectively) have been used. ${ }^{20,21}$ The AVTZ basis set has been employed in all geometry optimization and harmonic frequency calculations. Single point calculations using larger basis sets were performed at the ic-MRCI+Q/AVTZ equilibrium geometries and complete basis set (CBS) extrapolations were carried out using the $\mathrm{V} n \mathrm{Z}$ basis set series, with $n=\mathrm{Q}, 5$ and 6 . As in previous works, ${ }^{10,12,22}$ the CASSCF and dynamical correlation energies were extrapolated separately, using the $E_{\mathrm{CAS}}(\mathrm{CBS})+A \cdot \mathrm{e}^{-B \cdot n}$ and $E_{\mathrm{CAS}}(\mathrm{CBS})+C \cdot n^{-3}$ functionals, respectively. Such extrapolations led to the prediction of converged values of the energy difference properties (ionization and transition energies).

\section{B. Vibrational problem}

Harmonic vibrational frequencies were calculated by numerical differences using a homemade program interfaced with MOLPRO. It is dedicated to linear molecules and exploits the $\mathrm{C}_{2 \mathrm{v}}$ and $\mathrm{C}_{\mathrm{s}}$ abelian subgroup symmetries for linear and bent structures, respectively. These symmetries are explicitly taken into account in the ic-MRCI calculations, which lead to an important gain of computer time compared with the generic procedure implemented in MOLPRO for any kind of symmetry by setting the no-symmetry option $\left(\mathrm{C}_{1}\right.$ subgroup $)$ for multireference calculations. The block-diagonal form of the cartesian Hessian matrix also allows a separate diagonalization for the $\sigma$ and $\pi_{x}$ (or $\pi_{y}$ ) frequencies, and only one of the $\pi$ blocks needs to be calculated, as $\pi_{x}$ and $\pi_{y}$ frequencies are degenerate. Apart from computer time saving, the major advantage of this symmetry-adapted procedure is that it ensures the explicit separation of the $\mathrm{A}^{\prime}$ and $\mathrm{A}^{\prime \prime}$ Renner-Teller (RT) components of the $\Lambda \neq 0$ electronic states. In contrast, when the calculation is carried out without symmetry, the frequencies of the two RT-components are calculated from two successive roots of the Hamiltonian matrix. For a molecule having more than one vibrational coordinate which breaks the electronic degeneracy (a linear molecule larger than triatomic, for instance), it may happen that some of these coordinates stabilize the $\mathrm{A}^{\prime}$ component more than the $\mathrm{A}^{\prime \prime}$ one, while other ones produce the opposite effect. In such a case, finite differences determined from a given root of the Hamiltonian erroneously mix the energies of the $\mathrm{A}^{\prime}$ and $\mathrm{A}^{\prime \prime}$ PESs.

Such a situation occuring in the $\Pi$ and $\Phi$ excited states of $\mathrm{HC}_{5} \mathrm{~N}^{+}$motivated us to develop the symmetry-adapted code. We showed that calculations performed using $\mathrm{C}_{1}$ symmetry, as 
Publishimgthe standard MOLPRO code, led to incorrect results, with a mix-up of the $\pi$ frequencies arising from the $\mathrm{A}^{\prime}$ and $\mathrm{A}^{\prime \prime}$ electronic components, and deviations of up to $20 \mathrm{~cm}^{-1}$ of the individual frequency values.

Exploratory variational calculations using the Vibrational Mean Field Configuration Interaction approach (VMFCI) were also performed using the CONVIV program ${ }^{23}$ to highlight possible anharmonic couplings between vibrational modes.

\section{Spin-orbit coupling}

The spin-orbit $(\mathrm{SO})$ splittings of the $\Lambda \neq 0$ states have been determined by means of the state-interacting method, ${ }^{24}$ in which the Breit-Pauli spin-orbit Hamiltonian (BPSOH) is diagonalized in the basis of the non-relativistic $(\Lambda, S)$ wavefunctions of all the states calculated in this work. The non-diagonal matrix elements were calculated with the corresponding ic-MRCI wavefunctions, using the effective Hamiltonian approach implemented in MOLPRO. ${ }^{24}$ The diagonal matrix elements were replaced by the corresponding ic-MRCI+Q energies in order to improve the zeroth-order energies.

\section{Computer codes}

Apart from the MOLPRO program already referenced above, other computer codes have been used to analyze the results. Orbital isocontour maps and normal vibrational displacement vectors were drawn by means of the MOLDEN program. ${ }^{25}$ Franck-Condon spectrum simulations were carried out by means of the Pgopher software, using our ab initio results as input data. ${ }^{26}$

\section{RESULTS AND DISCUSSION}

\section{A. Electronic structure of $\mathrm{HC}_{5} \mathrm{~N} / \mathrm{HC}_{5} \mathrm{~N}^{+}$}

The ground state of neutral $\mathrm{HC}_{5} \mathrm{~N}$ arises from the closed-shell configuration:

- $(\alpha)[\ldots] 13 \sigma^{2} 1 \pi^{4} 2 \pi^{4} 3 \pi^{4}\left(\mathrm{X}^{1} \Sigma^{+}\right)$,

where $[\ldots]$ stands for a closed-shell core of twelve $\sigma$ MOs and $(\alpha)$ denotes the ground configuration of the neutral system. The configurations of the cation will be labelled by 
Publishimgnbers following their energy order, starting from the ground configuration (1), which results from the ejection of an electron from the HOMO of $(\alpha)$ :

- (1) $[\ldots] 13 \sigma^{2} 1 \pi^{4} 2 \pi^{4} 3 \pi^{3}\left(\mathrm{X}^{+2} \Pi\right)$.

Extracting an electron from the HOMO-1, HOMO-2 and HOMO-3 leads to the low-lying excited configurations, from which single spin-doublet electronic states arise:

- (2) $[\ldots] 13 \sigma^{2} 1 \pi^{4} 2 \pi^{3} 3 \pi^{4}\left({ }^{2} \Pi\right)$,

- (3) $[\ldots] 13 \sigma^{1} 1 \pi^{4} 2 \pi^{4} 3 \pi^{4}\left({ }^{2} \Sigma^{+}\right)$, and

- (4) $[\ldots] 13 \sigma^{2} 1 \pi^{3} 2 \pi^{4} 3 \pi^{4}\left({ }^{2} \Pi\right)$.

As already pointed out by Cao and Peyerimhoff ${ }^{5}$ and confirmed by our calculations, there is a fifth configuration close in energy to (4), leading to five excited states: ${ }^{27}$

- (5) [...] $13 \sigma^{2} 1 \pi^{4} 2 \pi^{4} 3 \pi^{2} 4 \pi^{1}\left({ }^{2} \Pi(\times 3) \oplus^{2} \Phi \oplus^{4} \Pi\right)$.

This configuration corresponds to a $3 \pi \rightarrow 4 \pi$ single excitation from (1), but, in the framework of the ionization process, it is a bielectronic excitation from $(\alpha)$. A total of $\operatorname{six}^{2} \Pi$ states thus arise from the low-lying configurations of the cation, which suggests, as confirmed by the results, that state mixing will occur and that avoided crossings will affect the corresponding PESs. The five MOs $(13 \sigma, 1 \pi, 2 \pi, 3 \pi$, and $4 \pi)$ govern the electronic structure of the cation and define the CAS (see Figure 1). Their shape will be useful for discussing the observed equilibrium properties of the calculated electronic states.

\section{B. Equilibrium properties}

\section{Characterization of the equilibrium structures}

Geometry optimizations were carried out at the ic-MRCI+Q/AVTZ level of theory on the PESs corresponding to all of the states arising from configurations $(\alpha)$ and (1) to (5). Eight stationary points were located and characterized on these PESs: two corresponding to the ground states of $\mathrm{HC}_{5} \mathrm{~N}$ and $\mathrm{HC}_{5} \mathrm{~N}^{+}$and seven to excited states of the cation. Let us note that no minimum has been found on the $5^{2} \Pi \mathrm{PES}$, as explained in section III E 1.The analysis of the ic-MRCI wavefunctions at equilibrium of each state provides the weights of 
\$13LE I. Analysis of the ic-MRCI wavefunctions of $\mathrm{HC}_{5} \mathrm{~N}$ and $\mathrm{HC}_{5} \mathrm{~N}^{+}$in terms of electronic configurations. $^{\text {a }}$

\begin{tabular}{|c|c|c|c|}
\hline System & State $^{\mathrm{b}}$ & Label $^{\mathrm{c}}$ & Weight $^{\mathrm{d}}$ \\
\hline $\mathrm{HC}_{5} \mathrm{~N}$ & $1^{1} \Sigma^{+}$ & $\mathrm{X}$ & $79 \%(\alpha)+4 \%(\beta)$ \\
\hline \multirow[t]{8}{*}{$\mathrm{HC}_{5} \mathrm{~N}^{+}$} & $1^{2} \Pi$ & $\mathrm{X}^{+}$ & $53 \%(1)+22 \%(2)$ \\
\hline & $2^{2} \Pi$ & $\mathrm{A}^{+}$ & $34 \%(2)+18 \%(1)+$ \\
\hline & $1^{2} \Sigma^{+}$ & $\mathrm{B}^{+}$ & $79 \%(3)+$ \\
\hline & $1^{4} \Pi$ & - & $74 \%(5)$ \\
\hline & $3^{2} \Pi\left(\operatorname{Min}_{1}\right)$ & - & $67 \%(5)$ \\
\hline & $3^{2} \Pi\left(\operatorname{Min}_{2}\right)$ & $\mathrm{C}^{+}$ & $62 \%(4)+11 \%(6)+7 \%(5)$ \\
\hline & $1^{2} \Phi$ & - & $73 \%(5)+3 \%(7)$ \\
\hline & $4^{2} \Pi$ & - & $71 \%(5)+3 \%(7)+3 \%(8)$ \\
\hline
\end{tabular}

${ }^{a}$ See text for the definition of the electronic configurations: (1) to (11) for $\mathrm{HC}_{5} \mathrm{~N}^{+},(\alpha)$ and $(\beta)$ for $\mathrm{HC}_{5} \mathrm{~N}$;

b States are listed in adiabatic energy ordér (see Tables V and VI), and numbered accordingly within each symmetry manifold;

${ }^{c}$ Spectroscopic labels (only for the experimentally observed states);

d Weights at the equilibrium geometries of the corresponding states. Only configurations with a contribution higher than $3 \%$ are considered.

the main contributing electronic configurations, as reported in Table I. The weight of a configuration (in \%) is calculated as the sum of squares of the contributing MRCI coefficients. A threshold of $3 \%$ is used for eliminating the less important configurations. A set of secondary configurations is adding up to the configurations list already presented:

- (6) $[\ldots] 13 \sigma^{2} 1 \pi^{4} 2 \pi^{3} 3 \pi^{3} 4 \pi^{1}$,

- (7) [... $13 \sigma^{2} 1 \pi^{4} 2 \pi^{2} 3 \pi^{4} 4 \pi^{1}$,

(8) $[\ldots] 13 \sigma^{2} 1 \pi^{3} 2 \pi^{3} 3 \pi^{3} 4 \pi^{2}$,

- (9) $[\ldots] 13 \sigma^{2} 1 \pi^{4} 2 \pi^{3} 3 \pi^{2} 4 \pi^{2}$,

- (10) $[\ldots] 13 \sigma^{1} 1 \pi^{4} 2 \pi^{4} 3 \pi^{3} 4 \pi^{1}$,

- (11) $[\ldots] 13 \sigma^{1} 1 \pi^{4} 2 \pi^{4} 3 \pi^{2} 4 \pi^{2}$, 
- $(\beta)[\ldots] 13 \sigma^{2} 1 \pi^{4} 2 \pi^{4} 3 \pi^{2} 4 \pi^{2}$

for $\mathrm{HC}_{5} \mathrm{~N}$.

The calculated states are listed by order of increasing adiabatic energies (see sections III C and III D 1). The second column of Table I gives the standard numbering of the states, by energy order for each orbital and spin symmetry. The third column provides the corresponding spectroscopic labels, introduced by Bieri et al. ${ }^{28}$ for $\mathrm{X}^{+}, \mathrm{A}^{+}, \mathrm{B}^{+}$, and $\mathrm{C}^{+}$. The multireference character of most states clearly appears when looking at the fourth column of the table, which reports the configuration weights. This character, already pointed out in previous works on cyanopolyyne cations, ${ }^{9-12}$ comes from the mixing between the $\pi$ orbitals forming such linear chains (see Fig. 1). The configuration mixing is important within the ${ }^{2} \Pi$ state manifold, as already postulated before. The competition between configurations (4) and (5) is of particular interest. It leads to the formation of two minima in the third PES of ${ }^{2} \Pi$ symmetry. These minima will be referred to as $3^{2} \Pi\left(\operatorname{Min}_{1}\right)$ and $3^{2} \Pi\left(\operatorname{Min}_{2}\right)$, respectively. As indicated in Table I, the latter minimum has been assigned to the $\mathrm{C}^{+}$state observed in the TPE spectrum, according to the successful Franck-Condon factor simulation presented in Paper I. ${ }^{1}$ We refer the reader to Figure 3 of Paper I and to the related text. This simulation reproduces very satisfactorily the vibrational progressions of the $\mathrm{X}^{+}, \mathrm{A}^{+}, \mathrm{B}^{+}$, and $\mathrm{C}^{+}$states, and, in the case of $\mathrm{C}^{+}$, it thus confirms the observation of the vibration within the $3^{2} \Pi\left(\mathrm{Min}_{2}\right)$ potential well. This assignment is furthermore consistent with the fact that, according to the electric dipole approximation, single-photon ionization implies a single electron excitation process. This is actually the case for the $(\alpha) \rightarrow(4) \mathrm{Min}_{2}$ excitation, unlike for the $(\alpha) \rightarrow(4) \operatorname{Min}_{1}$ excitation.

Let us note that the good agreement between the simulated and experimental spectra rests on a set of complementary ab initio results, detailed in the next sections:

(i) the ionization energy values, which give the energy positions of the 0-0 origin bands,

(ii) the vibrational frequency values of the cationic states, which predict the energy spacings within the progression series with respect to the 0-0 origin, and

(iii) the FCF values, which provide relative values of the band intensities. 
Publishifige latter quantities require the calculation of the vibrational overlap integrals between the neutral ground state and the cationic states of interest, taking the Dushinski rotation matrix into account. These integrals depend on the calculated geometries and on the harmonic eigensolutions of both states. The use of the harmonic approximation has been found sufficient for an unambiguous identification of most of the observed vibrational features. Larger deviations in the predicted intensities of the $\mathrm{C}^{+}$states bring to light the existence of vibronic couplings induced by the double well shape of the $3^{2} \Pi$ PES, as discussed in section III 5 .

\section{Geometries and rotational constants}

The equilibrium properties (bond distances and rotational constants $B_{\mathrm{e}}$ ) have been calculated at the ic-MRCI+Q/AVTZ level for all stationary points. The results are reported in Table II. We refer to (1) in section I for the definition of the atom numbering.

As confirmed by the calculated vibrational frequencies (see next section), the global minima of all calculated states correspond to linear geometries, except for the $1^{4} \Pi$ state, for which the linear form is a local minimum. This state is indeed split into a couple of RennerTeller planar bent states $\left(1^{4} \mathrm{~A}^{\prime}\right.$ and $\left.1^{4} \mathrm{~A}^{\prime \prime}\right)$, among which the ${ }^{4} \mathrm{~A}^{\prime \prime}$ component is the global minimum, as detailed in section IIIB 3. The geometries of the local and global minima are both reported in Table II.

The results are compared with the available theoretical and experimental values from the literature, but as it is the first time that the equilibrium properties of the excited states of the cation are calculated, the comparison is limited to the ground state properties.

The ic-MRCI $+\mathrm{Q}$ results are in good agreement with the available experimental values, noting howevêr that theoretical values are equilibrium properties while experimental ones are $v=0$ vibrationally averaged ones. For the ground state of $\mathrm{HC}_{5} \mathrm{~N}$, the deviation with the microwave spectroscopy results ${ }^{31}$ is $0.3 \%$ for the rotational constant and $<0.9 \%$ for the

bond distances. For the cation, the deviation of the calculated rotational constants with respect to the frequency modulation spectroscopy values ${ }^{32}$ is $0.3 \%$ and $0.02 \%$ for the $\mathrm{X}^{+}$ and $\mathrm{A}^{+}$states, respectively.

On the theoretical side, the results on the neutral ground state can be compared with $\operatorname{CCSD}(\mathrm{T})$ calculations. Deviations of our calculated ic-MRCI bond distances with the VTZ, VQZ and VTZ corr CCSD(T) results $^{29,30}$ are $<1 \%,<0.8 \%$ and $<0.5 \%$, respectively. 
state of $\mathrm{HC}_{5} \mathrm{~N}$ and of the low-lying states of $\mathrm{HC}_{5} \mathrm{~N}^{+}$.

\begin{tabular}{|c|c|c|c|c|c|c|c|c|}
\hline \multicolumn{9}{|c|}{$\mathrm{HC}_{5} \mathrm{~N}$} \\
\hline State & Method $^{\mathrm{a}}$ & $\mathrm{H}-\mathrm{C}_{1}$ & $\mathrm{C}_{1} \equiv \mathrm{C}_{2}$ & $\mathrm{C}_{2}-\mathrm{C}_{3}$ & $\mathrm{C}_{3} \equiv \mathrm{C}_{4}$ & $\mathrm{C}_{4}-\mathrm{C}_{5}$ & $\mathrm{C}_{5} \equiv \mathrm{N}$ & $B_{\mathrm{e}}$ \\
\hline \multirow[t]{5}{*}{$\mathrm{X}^{1} \Sigma^{+}$} & & 1.061 & 1.203 & 1.371 & 1.215 & 1.376 & 1.155 & 1327.1 \\
\hline & $\operatorname{CCSD}(\mathrm{T}) / \mathrm{VTZ}^{\mathrm{b}}$ & 1.064 & 1.216 & 1.372 & 1.219 & 1.377 & 1.168 & 1317.1 \\
\hline & $\operatorname{CCSD}(\mathrm{T}) / \mathrm{VTZ}$ corr. ${ }^{\mathrm{a}}$ & 1.063 & 1.209 & 1.366 & 1.213 & 1.371 & 1.161 & 1330.1 \\
\hline & $\operatorname{CCSD}(\mathrm{T}) / \mathrm{VQZ}^{\mathrm{c}}$ & 1.064 & 1.212 & 1.370 & & 1.376 & 1.165 & - \\
\hline & Exp. $^{\mathrm{d}}$ & 1.0569 & 1.2087 & 1 & & 1.3636 & 1.1606 & 1331.3313 \\
\hline \multicolumn{9}{|c|}{$\mathrm{HC}_{5} \mathrm{~N}^{+}$} \\
\hline \multirow[t]{5}{*}{$\mathrm{X}^{+2} \Pi$} & & 1.0712 & 1.234 & 1.317 & 1.229 & 1.352 & 1.174 & 1341.0 \\
\hline & UB3LYP /TZVP & 1.07 & 1.23 & 1.32 & 1.24 & 1.34 & 1.17 & - \\
\hline & UBLYP $/ 6-311 \mathrm{G}^{* * \mathrm{f}}$ & 1.078 & 1.240 & 1.325 & 1.251 & 1.339 & 1.188 & 1325.8 \\
\hline & B3LYP /6-311G* g & 1.07 & & 1.322 & 1.239 & 1.338 & 1.173 & 1342.4 \\
\hline & Exp.h & & & & - & - & - & 1337.56 \\
\hline \multirow[t]{2}{*}{$\mathrm{A}^{+2} \Pi$} & & & & 1.383 & 1.216 & 1.342 & 1.202 & 1314.8 \\
\hline & Exp. ${ }^{\mathrm{g}}$ & & & - & - & - & - & 1314.55 \\
\hline $\mathrm{B}^{+2} \Sigma^{+}$ & & & & 1.351 & 1.216 & 1.343 & 1.151 & 1349.2 \\
\hline $1^{4} \Pi$ & & & 1.273 & 1.263 & 1.329 & 1.273 & 1.221 & 1322.2 \\
\hline $1^{4} \mathrm{~A}^{\prime \prime}$ planar bent ${ }^{\mathrm{i}}$ & & & 1.324 & 1.247 & 1.316 & 1.299 & 1.192 & $1342.8,1338.2^{j}$ \\
\hline $3^{2} \Pi\left(\operatorname{Min}_{1}\right)$ & & & 1.261 & 1.279 & 1.319 & 1.292 & 1.207 & 1321.7 \\
\hline $3^{2} \Pi\left(\operatorname{Min}_{2}\right)\left(\mathrm{C}^{+}\right)$ & & & 1.212 & 1.380 & 1.241 & 1.381 & 1.199 & 1292.9 \\
\hline $1^{2} \Phi$ & & & 1.271 & 1.265 & 1.330 & 1.283 & 1.211 & 1322.6 \\
\hline $4^{2} \Pi$ & & 1.070 & 1.266 & 1.273 & 1.327 & 1.285 & 1.212 & 1320.4 \\
\hline
\end{tabular}

a This work, unless specified, from ic-MRCI+Q/AVTZ calculations;

b From Ref. 29 ; CCSD(T)/VTZ corr values are corrected for systematic errors in similar systems;

${ }^{c}$ From Ref. 30;

${ }^{\mathrm{d}}$ Microwave spectroscopy ${ }^{31} B_{0}$ and $r_{0}$ from a substitution structure;

e From Ref. 3

${ }^{\mathrm{f}}$ From Ref. 2 ;

g From Ref. 5;

${ }^{\text {h }}$ Frequency modulation absorption spectroscopy; ${ }^{32} B_{0}$ value;

${ }^{i}$ Renner-Teller component of the $1^{4} \Pi$ state (see section III.B.3). The values of the bending angles are $140,-176,180,-165$, and 175 degrees, for the $\angle \mathrm{HC}_{1} \mathrm{C}_{2}, \angle \mathrm{C}_{1} \mathrm{C}_{2} \mathrm{C}_{3}, \angle \mathrm{C}_{2} \mathrm{C}_{3} \mathrm{C}_{4}, \angle \mathrm{C}_{3} \mathrm{C}_{4} \mathrm{C}_{5}$, and $\angle \mathrm{C}_{4} \mathrm{C}_{5} \mathrm{~N}$ angles, respectively.

${ }^{\mathrm{j}} B \simeq C$ rotational constants corresponding to rotation about the inertial axes perpendicular to the quasi-linear frame. $\mathrm{b}$ lies within the molecular plane and $\mathrm{c}$ is perpendicular to it. The value of $A$ is $400039 \mathrm{MHz}$. 
Publishínge VTZ corr values involve a correction for systematic overestimates of the bond distances evaluated from similar systems using $\operatorname{CCSD}(\mathrm{T})$. Our ic-MRCI+Q results are in better agreement with these corrected values. We pointed out similar discrepancies resulting from the monoreference correlation treatment of the $\operatorname{CCSD}(\mathrm{T})$ approach in our paper on $\mathrm{HC}_{3} \mathrm{~N}^{+} .{ }^{10}$ This shows again that multiconfigurational effects are important for representing the $\pi$ electron delocalization along the molecular chain.

For the ground state of the cation, the deviations with the DFT results are larger but still reasonable $\left(<0.9 \%,<1.8 \%\right.$, and $<1 \%$ with UB3LYP/TZVP ${ }^{3}$ UBLYP $/ 6-311 \mathrm{G}^{* *}{ }^{2}$ and B3LYP $/ \mathrm{DZVP}^{5},{ }^{5}$ respectively).

Bond lengths are found to vary significantly (up to 9.5\%) from one state to another. As in the case of $\mathrm{HC}_{3} \mathrm{~N}^{+},{ }^{10}$ the changes can be rationalized in terms of the main configuration changes reported in Table I and of the bonding/antibonding characters of the involved MOs (see Fig. 1). Ejecting an electron from a given MO induces indeed a weakening of its features (bonding or antibonding) and accordingly a change of the corresponding bond lengths (extension or contraction, respectively). The relative magnitude of these effects on the different bonds were verified by an analysis of the overlap Mulliken atomic populations. The following trends, already pointed out for $\mathrm{X}^{+}$and $\mathrm{A}^{+},{ }^{32}$ can be drawn for the ionization towards different states of the cation:

(i) $\mathrm{X} \rightarrow \mathrm{X}^{+}$: an electron is ejected from $3 \pi$, which exhibits bonding triple bonds and antibonding single bonds (see Fig. 1), with as a result an extension of the formers and a contraction of the latters. The rotational constant value increases by $1 \%$ because the contractions are globally larger than the extensions.

(ii) $\mathrm{X} \rightarrow \mathrm{A}^{+}$: the bonding regions of $2 \pi$, which lose an electron, cover the $\mathrm{C}_{1} \mathrm{C}_{2}, \mathrm{C}_{2} \mathrm{C}_{3}$, and $\mathrm{C}_{5} \mathrm{~N}$ bonds, which are stretched $\left(4 \%\right.$ for $\left.\mathrm{C}_{5} \mathrm{~N}\right)$, while the $\mathrm{C}_{4} \mathrm{C}_{5}$ bond contracts. The net result is an elongation of the structure, with a $0.9 \%$ decrease of the $B_{\mathrm{e}}$ value.

(iii) $\mathrm{X} \rightarrow \mathrm{C}^{+}\left(=3^{2} \Pi\left(\mathrm{Min}_{2}\right)\right)$ : the electron ejected from the fully bonding $1 \pi \mathrm{MO}$ induces a stretch of all bonds, in particular of $\mathrm{C}_{3} \mathrm{C}_{4}(2 \%)$ and $\mathrm{C}_{5} \mathrm{~N}(4 \%)$. The $B_{\mathrm{e}}$ value decreases consequently by $2.6 \%$.

(iv) $\mathrm{X} \rightarrow 3^{2} \Pi\left(\mathrm{Min}_{1}\right), 1^{2} \Phi$ and $4^{2} \Pi$ : spectacular geometry changes characterize all states arising from configuration (5), as a result of the superposition of two effects: the loss of 
a $3 \pi$ electron and a $3 \pi \rightarrow 4 \pi$ excitation. All states obey to the same trends: increase of all triple bond lengths (by more than $5 \%, 9 \%$, and $5 \%$ for $\mathrm{C}_{1} \mathrm{C}_{2}, \mathrm{C}_{3} \mathrm{C}_{4}$, and $\mathrm{C}_{5} \mathrm{~N}$, respectively) and decrease of the single bond lengths (by more than 7\%). Due to a compensation of both opposite effects, the net extension of the structures is small, and the $B_{\mathrm{e}}$ value decreases by less than $0.5 \%$.

The non-planar geometry of the ${ }^{4} \mathrm{~A}^{\prime \prime}$ state, reported in Table II (see footnotes), corresponds to a quasi-linear structure, as confirmed by the large value of the $A$ rotational constant related to the rotation about the inertial axis a which lies along the molecular frame. The values of the equilibrium bending angles characterize the zig-zag shape of the chain, presenting an alternation of positive and negative angle values. The main deviation from linearity comes from the $\mathrm{HC}_{1} \mathrm{C}_{2}$ bending of 140 degrees, and to a lesser extent from the $\mathrm{C}_{3} \mathrm{C}_{4} \mathrm{C}_{5}$ bending of 165 degrees. The fact that the rotational constants $B \simeq C$ characterizes moreover a near prolate asymmetric top.

\section{Vibrational frequencies}

Table III reports the results of harmonic vibrational frequencies calculated at the icMRCI+Q/AVTZ level of theory using the symmetry adapted procedure explained in section II B. The calculations were performed at the linear equilibrium geometries of all states. The normal coordinate displacements of the bending frequencies of the four low-lying states of the cation are depicted in Figure 2, while those of the stretching frequencies were already shown in Paper $I^{1}$ to illustrate the vibrational signatures of our TPE spectrum. Apart from the differences mentioned in Paper I between the stretching normal modes of the considered states, one observes that the bending coordinates are quite similar in all the considered states, except for the $\mathrm{B}^{+}$state, in which $\nu_{7}, \nu_{8}$, and $\nu_{9}$ correspond to quasi pure $\mathrm{C}_{4} \mathrm{C}_{5} \mathrm{~N}$, $\mathrm{HC}_{1} \mathrm{C}_{2}$ and $\mathrm{C}_{2} \mathrm{C}_{3} \mathrm{C}_{4}$ local bendings, respectively, while in the other states they mainly contribute to $\nu_{9}, \nu_{7}$, and $\nu_{8}$, respectively. We also note that the frequencies of $\mathrm{B}^{+}$are globally higher than those of the other states, as indicated by the high value of the ZPE. This is a consequence of the strengthening of the $\pi$ structure by the three closed $\pi$ MOs.

As already pointed out, the linearity characterizes the global minima of all states expect the $1^{4} \Pi$ state. This is confirmed by the real frequencies calculated for all modes in the case of linear global minima. In the case of the $1^{4} \Pi$ state, an imaginary frequency, marked in 
This manuscript was accepted by J. Chem. Phys. Click here to see the version of record.

TABLE III. Calculated harmonic vibrational frequencies (in $\mathrm{cm}^{-1}$ ) of $\mathrm{HC}_{5} \mathrm{~N}^{+}$. a

\begin{tabular}{|c|c|c|c|c|c|c|c|c|}
\hline & $\mathrm{X}^{+2} \mathrm{\Pi}$ & $\mathrm{A}^{+2} \Pi$ & $\mathrm{B}^{+2} \Sigma^{+}$ & $1^{4} \Pi$ & $3^{2} \Pi\left(\operatorname{Min}_{1}\right)$ & $3^{2} \Pi\left(\operatorname{Min}_{2}\right)\left(\mathrm{C}^{+}\right)$ & $1^{2} \Phi$ & $4^{2} \Pi$ \\
\hline$\nu_{1}(\sigma)$ & 3403 & 3412 & 3456 & 3407 & 3397 & 3433 & 3388 & 3393 \\
\hline$\nu_{2}(\sigma)$ & 2300 & 2350 & 2339 & 2339 & 2742 & 2214 & 2208 & 2278 \\
\hline$\nu_{3}(\sigma)$ & 2121 & 2210 & 2282 & 1937 & 1841 & 2170 & 1810 & 1829 \\
\hline$\nu_{4}(\sigma)$ & 1985 & 1999 & 2141 & 1507 & 1637 & 10 & 1605 & 1667 \\
\hline$\nu_{5}(\sigma)$ & 1238 & 1097 & 1244 & 1282 & 1296 & & 1308 & 1307 \\
\hline$\nu_{6}(\sigma)$ & 639 & 618 & 654 & 485 & 636 & & 641 & 640 \\
\hline$\mu-(\pi)^{b}$ & $(803,693)$ & $(695,705)$ & & $(775,296 \mathrm{i})^{\mathrm{c}}$ & $(772,411)$ & & $(550,550)$ & $(414,821)$ \\
\hline$\nu_{7}(\pi)^{\mathrm{D}}$ & {$[750,0.15]$} & {$[700,-0.02]$} & 847 & $-d$ & {$[618,0.56]$} & & {$[550,0.00]$} & {$[650,-0.60$} \\
\hline$\mu^{2}(\pi)^{\mathrm{b}}$ & $(607,546)$ & $(554,545)$ & & $(586,386)$ & $(555,375)$ & & $(491,491)$ & $(398,605)$ \\
\hline$\nu_{8}(\pi)^{\circ}$ & {$[577,0.11]$} & {$[549,0.02]$} & 776 & {$[496,0.39]$} & {$\left[\begin{array}{lll}474 & 0 & 37\end{array}\right]$} & & {$[491,0.00]$} & {$[512,-0.40$} \\
\hline & $(446,468)$ & $(476,475)$ & & $(472,353)$ & $(462,315)$ & $(457,446)$ & $(423,423)$ & $(249,437)$ \\
\hline$\nu_{9}(\pi)^{\mathrm{b}}$ & {$[457,-0.05]$} & {$[476,0.0]$} & 566 & {$[417,0.28]$} & {$[396,0.37]$} & {$[452,0.03]$} & {$[423,0.00]$} & {$[356,-0.51$} \\
\hline & $(260,261)$ & $(277,277)$ & & $(270,285)$ & & $(270,262)$ & $(281,281)$ & $(228,264)$ \\
\hline$\nu_{10}$ & {$[261,0.00]$} & {$[277,0.00]$} & 264 & {$[277,-0.06]$} & & {$[266,0.03]$} & {$[281,0.00]$} & {$[247,-0.15$} \\
\hline & $(106,110)$ & $(104,107)$ & & $(118,108)$ & 3,71 & $(112,113)$ & $(97,97)$ & $(70,102)$ \\
\hline$\nu_{11}(\pi)^{\mathrm{b}}$ & {$[108,-0.04]$} & {$[105,-0.03]$} & 106 & {$[113,0.09]$} & $8,0.2$ & {$[112,0.00]$} & {$[97,0.00]$} & {$[88,-0.36]$} \\
\hline $\mathrm{ZPE}^{\mathrm{e}}$ & 7996 & 7950 & 8617 & & & 7994 & 7323 & 7409 \\
\hline
\end{tabular}

${ }^{\text {a }}$ Calculations performed at the ic-MRCI+Q/AVTZ level;

${ }^{\mathrm{b}}$ Modes of $\pi$ symmetry of the $\Pi$ and $\Phi$ electronic states are characterized by two different frequencies $\left(\omega\left(\mathrm{A}^{\prime}\right), \omega\left(\mathrm{A}^{\prime \prime}\right)\right)$ corresponding to the $\mathrm{A}^{\prime}$ and $\mathrm{A}^{\prime \prime}$ Renner-Teller components, respectively. The numbers between squared brackets correspond to zeroth-order RT bending frequency $\omega_{0}$ and Renner parameter $\epsilon$, respectively (see text);

c The frequency of the $\mathrm{A}^{\prime \prime} \mathrm{RT}$ component is imaginary;

d Not calculated for a linear-bent RT effect;

e ZPE calculated using the $\omega_{0}$ values for the bending modes of the $\Lambda \neq 0$ states.

bold, indicates that the Renner-Teller (RT) splitting induces the non-linearity of one of its components, as detailed below.

The RT effect has been characterized for all the $\Lambda \neq 0$ states ( $\Pi$ and $\Phi$ ) for which the two-fold degeneracy is lifted upon planar bending, according to the $\mathrm{C}_{\infty \mathrm{v}} \rightarrow \mathrm{C}_{\mathrm{s}}$ symmetry correlation rule: $\Pi$ or $\Phi \rightarrow \mathrm{A}^{\prime} \oplus \mathrm{A}^{\prime \prime}$. The resulting pair of states will be referred to as a RT pair below.

The frequencies have been calculated independently for each component of the RT pairs using our symmetry-adapted procedure. For the stretching modes $\left(\nu_{1}\right.$ to $\left.\nu_{6}\right)$, belonging to the $\sigma$ symmetry, both electronic components remain degenerate and are characterized by the same frequency reported in Table III. On the contrary, the bending modes $\left(\nu_{7}\right.$ to $\left.\nu_{11}\right)$, of 


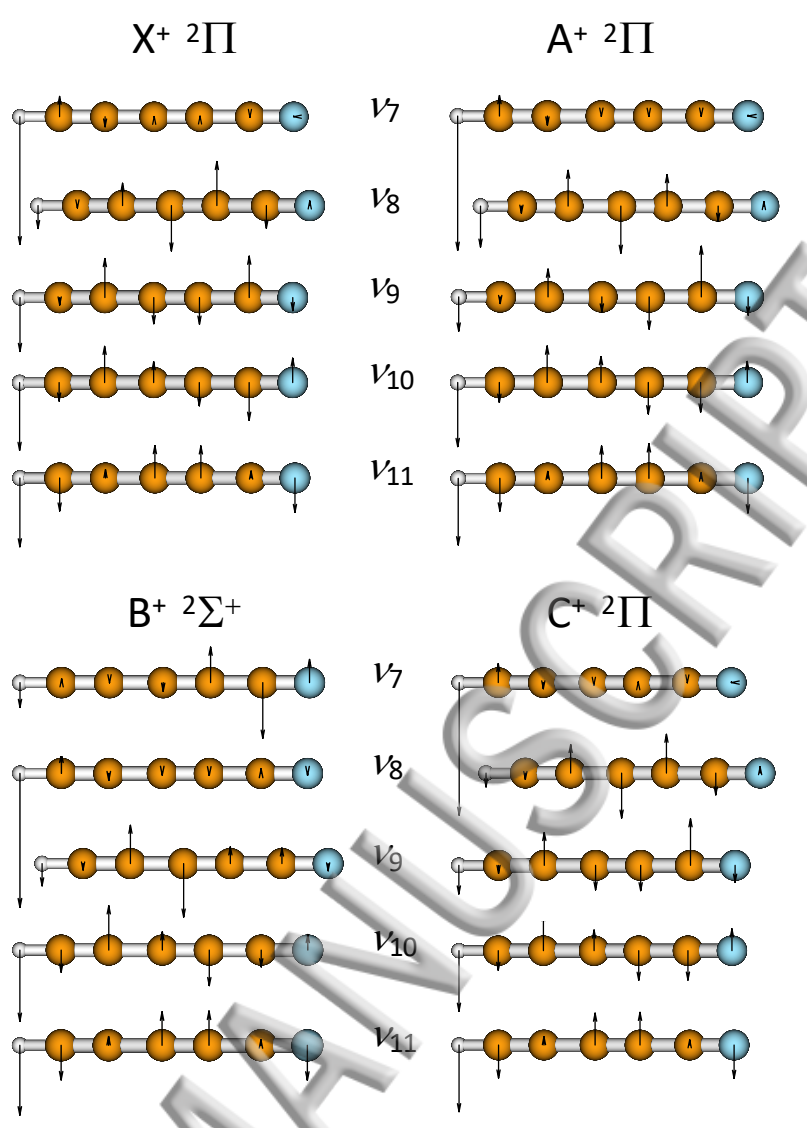

FIG. 2. Mass-weighted normal coordinates of the bending modes of the $\mathrm{X}^{+}, \mathrm{A}^{+}, \mathrm{B}^{+}$and $\mathrm{C}^{+}$states of $\mathrm{HC}_{5} \mathrm{~N}^{+}$, calculated at the ic-MRCI+Q/AVTZ level of theory. The corresponding frequencies are reported in Table III. Atomic displacements are scaled by a factor of 0.5 for the $\mathrm{CH}$-bending modes and by a factor of 0.8 for the other modes.

$\pi$ symmetry, are lifting the degeneracy, and the RT components $\mathrm{A}^{\prime}$ and $\mathrm{A}^{\prime \prime}$ are characterized by different frequencies reported as $\left(\omega\left(\mathrm{A}^{\prime}\right), \omega\left(\mathrm{A}^{\prime \prime}\right)\right)$ in the table. One observes that all ${ }^{2} \Pi$ and ${ }^{2} \Phi$ states are characterized by pairs of real frequencies and thus belong to the linearlinear RT case of splitting. This means that both PESs of the RT-pair $\left({ }^{2} \mathrm{~A}^{\prime}\right.$ and $\left.{ }^{2} \mathrm{~A}^{\prime \prime}\right)$ have a minimum at linear geometry.

In contrast, a linear-bent RT splitting occurs in the case of the ${ }^{4} \Pi$ state, for which all modes have real frequencies except the $\omega\left(\mathrm{A}^{\prime \prime}\right)$ frequency of $\nu_{7}$, which is imaginary. The global minimum of the ${ }^{4} \mathrm{~A}^{\prime} \mathrm{PES}$ is thus a linear structure, while the one of the ${ }^{4} \mathrm{~A}^{\prime \prime} \mathrm{PES}$ corresponds to a planar bent structure located in the direction of the $\nu_{7}\left(\mathrm{~A}^{\prime \prime}\right)$ normal coordinate. As for most of the calculated states (see Figure 2), $\nu_{7}$ mainly corresponds to the $\mathrm{HC}_{1} \mathrm{C}_{2}$ bending 
Publishimg ion. This is confirmed by the equilibrium value of this angle (140 degrees) reported in Table II for the bent equilibrium geometry of this state. The linear geometry of the ${ }^{4} \Pi$ state is a saddle point on the ${ }^{4} \mathrm{~A}^{\prime \prime} \mathrm{PES}$, calculated at the ic-MRCI+Q/AVTZ level to be $573 \mathrm{~cm}^{-1}$ higher in energy than the planar bent minimum.

The strength of the RT effect can easily be evaluated in the case of the linear-linear RT splitting that affects the ${ }^{2} \Pi$ and ${ }^{2} \Phi$ states. A first indication is provided by the magnitude of the frequency difference in a RT pair, but the $\epsilon$ parameter is a better indicator of this strength. It has been evaluated, as in our work on $\mathrm{HC}_{3} \mathrm{~N}^{+},{ }^{10}$ by means of an harmonic single-mode vibronic approach. ${ }^{27}$ This simplified method, negleeting anharmonicity and inter-mode vibronic coupling provides for each mode the zeroth-order bending frequency $\omega_{0}$ and the $\epsilon$ parameter. Both values are reported between square brackets in Table III. A comparison of the absolute values of $\epsilon$ in the different states shows that the RT effect is very weak for the $\mathrm{A}^{+}, \mathrm{C}^{+}\left(3^{2} \Pi\left(\mathrm{Min}_{2}\right)\right)$, and $1^{2} \Phi$ states. For the latter state, it is even negligible $(\epsilon<0.01)$. It is also weak for the $\mathrm{X}^{+}$state, except for the $\nu_{7}$ and $\nu_{8}$ modes. In contrast, it is strong for $3^{2} \Pi\left(\operatorname{Min}_{1}\right)$ and $4^{2} \Pi$ states that lie in a perturbed region. It is also important to note the occurence of positive and negative values of $\epsilon$. Its sign depends on the energy order of the ${ }^{2} \mathrm{~A}^{\prime}$ and ${ }^{2} \mathrm{~A}^{\prime \prime}$ states in the RT splitting: a positive value indicates that the energy of the ${ }^{2} \mathrm{~A}^{\prime \prime} \mathrm{PES}$ is more stable than the one of the ${ }^{2} \mathrm{~A}^{\prime} \mathrm{PES}$, or in other words that $\omega\left({ }^{2} \mathrm{~A}^{\prime}\right)>\omega\left({ }^{2} \mathrm{~A}^{\prime \prime}\right)$. The opposite is true when $\epsilon$ is negative, respectively. The occurrence of positive and negative values of $\epsilon$ for different modes of the same state is observed here for all states except for $4^{2} \Pi$. This implies that the use of a symmetry-adapted calculation of the frequencies is mandatory, as done here.

The comparison of the stretching frequencies of Table III with those extracted from our TPE spectrum has already been discussed in Paper $\mathrm{I}^{1}{ }^{1}$ where a Franck-Condon factor (FCF) simulation of the spectrum has facilitated the identification despite the harmonic approximation (see section III.B and Figure 3 of Ref. 1). The neglect of anharmonicity implies that most of the calculated values overestimate the experimental values, but the discrepancies are smaller than $30 \mathrm{~cm}^{-1}$ for most of the observed modes, except for $\nu_{2}$ and $\nu_{4}$ for which they reach $120 \mathrm{~cm}^{-1}$, noting however the uncertainty of $80 \mathrm{~cm}^{-1}$ on the TPES values. The existence of stronger anharmonic effects is likely for these modes, considering that they form with $\nu_{3}$ a cluster of close lying harmonic levels around $2000 \mathrm{~cm}^{-1}$, which 
Publishingrg involved in combinations of coupled bond stretching vibrations, as shown by the normal coordinates pictures depicted in Figure 1 of Ref. 1. This point has been investigated in the case of the $\mathrm{X}^{+}$state by means of 2 - and 3-mode variational calculations performed using the CONVIV program. ${ }^{23}$ They actually show qualitatively that the anharmonic coupling is important, mainly between $\nu_{2}$ and $\nu_{4}$, which may explain the larger discrepancies observed for $\nu_{2}$ but not for $\nu_{3}$.

Previous theoretical works only provide the harmonic frequencies of the $\mathrm{X}^{+}$state calculated with UBLYP/6-311G ${ }^{* * 2}$ and B3LYP/6-311G*5. The former works, limited to the stretching frequencies, are in excellent agreement (within $10 \mathrm{~cm}^{-1}$ ) with the experimental findings, although they did not include anharmonic corrections. The latter ones are closer to our ic-MRCI+Q values. Note however that the bending frequencies calculated with DFT faced with the symmetry issue of the RT components and cannot be compared to our symmetry adapted results.

\section{Electric dipole moments}

The electric dipole moment of linear molecular chains is aligned along the internuclear $Z$-axis and its projection $\mu_{\mathrm{e} z}$ on this axis is reported for all states in Table IV. This quantity provides two interesting classes of information: its absolute value gives the modulus of the permanent electric dipole moment vector, while its sign indicates how the electronic charges are distributed along the molecular chain, noting that the HCCCCCN nuclear frame is oriented toward positive $Z$ values. As the dipole moment of a charged system depends on the origin of the coordinate system, we must specify that the calculations are performed with the centre of mass as the coordinate origin.

All $A \neq 0$ states $\left({ }^{2} \Pi,{ }^{2} \Phi\right.$, and $\left.{ }^{4} \Pi\right)$ are characterized by a large negative value of $\mu_{\mathrm{e} z}$ of

about $-6.5 \mathrm{D}$, while the ${ }^{2} \Sigma^{+}$state has a smaller value of opposite sign $(+2.4 \mathrm{D})$. Similar results were obtained for $\mathrm{HC}_{3} \mathrm{~N}^{+},{ }^{10}$ and the interpretation is the same. The $13 \sigma$ orbital (9o in $\mathrm{HC}_{3} \mathrm{~N}^{+}$) is localized on the $\mathrm{CN} \sigma$ bond and on the nitrogen lone pair (see Fig. 1), which results in a large negative contribution to the dipole moment value. In contrast the delocalized $\pi$ MOs give smaller positive values, and the sign of $\mu_{\mathrm{e} z}$ thus results from the compensation between these opposite sign effects. 
\$13LE IV. Calculated equilibrium properties of the low-lying states of $\mathrm{HC}_{5} \mathrm{~N}^{+}$: electric dipole moment (in D) and spin-orbit constant $A_{\mathrm{SO}}\left(\right.$ in $\mathrm{cm}^{-1}$ ); this work, unless specified.

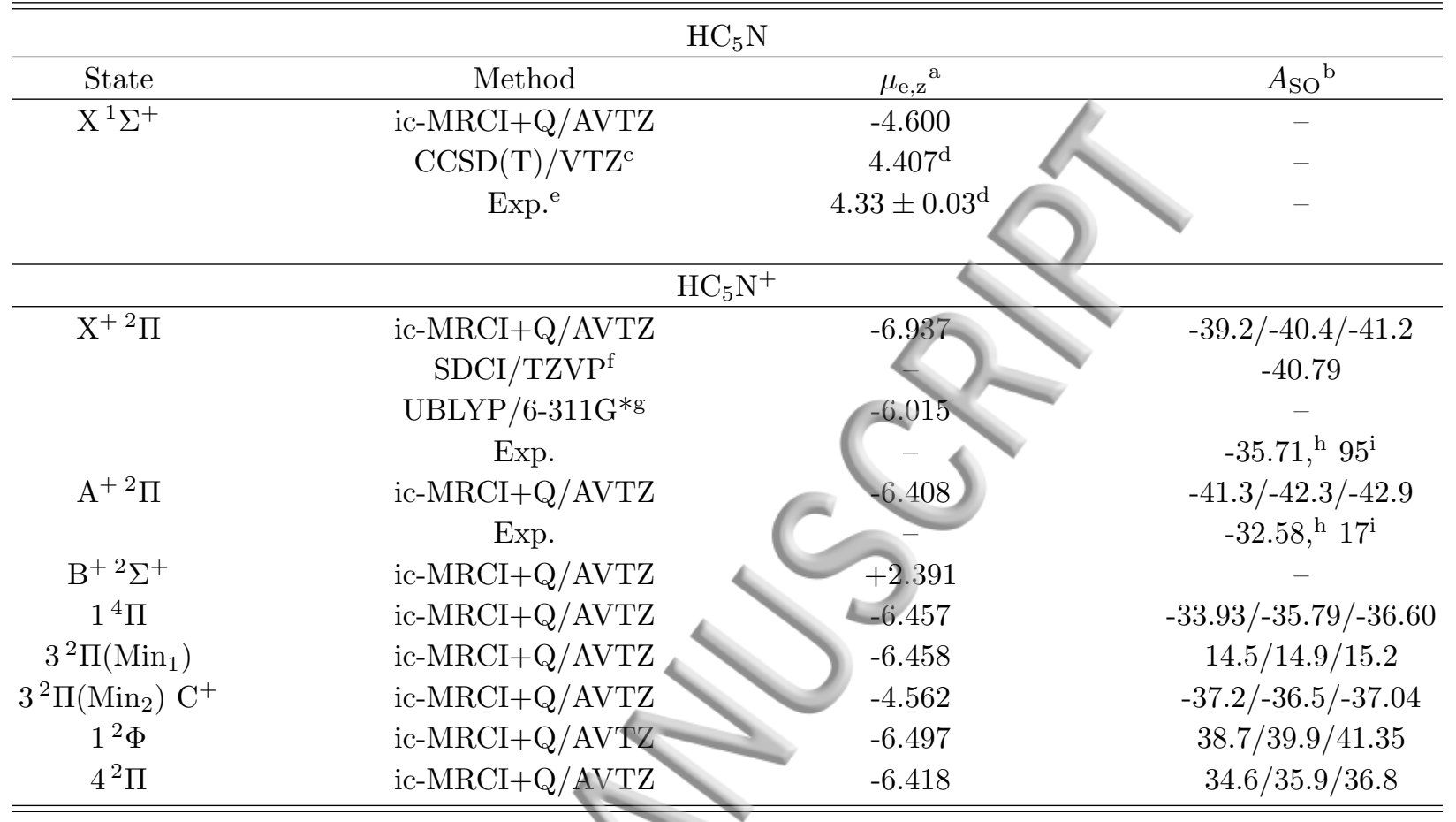

${ }^{a}$ Projection of the permanent electric dipole moment vector $\mu_{\mathrm{e}}$ along the internuclear axis $(\mathrm{OZ})$;

b This work, otherwise specified; calculated using BPSOH/ic-MRCI and VTZ/VQZ/V5Z basis sets.

Positive and negative signs of $A_{\mathrm{SO}}$ indicate regular and inverted splitting patterns, respectively;

${ }^{\text {c }}$ From Ref. 29;

d Modulus of $\mu_{\mathrm{e}}$;

e Microwave spectroscopy; ${ }^{31}$

${ }^{\mathrm{f}}$ From Ref. 3 ; spin-orbit calculation performed at UB3LYP/TZVP geometry using the full one- and two-electron no-pair spin-orbit Hamiltonian;

g From Ref. 2;

h Frequency/modulation absorption spectroscopy; ${ }^{32}$

${ }^{i}$ Laser induced/fluorescence in isolated matrix. ${ }^{33}$

\section{Spin-orbit constants}

Spin-orbit $A_{\mathrm{SO}}$ constants of the $\Pi$ and $\Phi$ states have been estimated by the spin-orbit splitting of their $\Omega$ components, calculated at the ic-MRCI+Q/AVTZ equilibrium geometries. For that purpose, the $30 \times 30 \mathrm{BPSOH}$ matrix involving the SO-components of the 7 lowest electronic states of the cation (four ${ }^{2} \Pi$, one ${ }^{2} \Phi$, one ${ }^{2} \Sigma^{+}$, and one ${ }^{4} \Pi$ states) has been diagonalized, as explained in section II C. The calculations were performed using the 
Publishibg' basis sets. The $A_{\mathrm{SO}}$ values converge to within less than $1 \mathrm{~cm}^{-1}$ along the $\mathrm{V} n \mathrm{Z}$ series $(n=\mathrm{T}, \mathrm{Q}, 5)$, as shown in Table IV, where the results are reported. The sign of the constant indicates a regular $\left(A_{\mathrm{SO}}>0\right)$ or inverted $\left(A_{\mathrm{SO}}<0\right)$ splitting of the ${ }^{2} \Pi$ and ${ }^{2} \Phi$ states. As expected, all states arising from a $\pi^{3}$ configurations $\left(\mathrm{X}^{+}, \mathrm{A}^{+}\right.$, and $\left.\mathrm{C}^{+}\right)$are inverted. States arising from configuration (5) are regular, except the ${ }^{4} \Pi$ state which is inverted. The order of magnitude of the splitting is almost the same for all states (between 37 and $42 \mathrm{~cm}^{-1}$ ), except the $3^{2} \Pi\left(\operatorname{Min}_{1}\right)$ value, which is only $15 \mathrm{~cm}^{-1}$. Our $\mathrm{X}^{+}$state value compares very well (within $0.4 \mathrm{~cm}^{-1}$ ) with the theoretical value of Tatchen et al., ${ }^{3}$ obtained using the full no-pair spin-orbit Hamiltonian and the SDCI/TZVP level of calculation. However, the deviations from the experimental values of Sinclair et al..$^{32}$ are larger $\left(5 \mathrm{~cm}^{-1}\right.$ for $\mathrm{X}^{+}$and $7 \mathrm{~cm}^{-1}$ for $\mathrm{A}^{+}$). Smith et al. ${ }^{33}$ also derived $A_{\mathrm{SO}}$ values for $\mathrm{X}^{+}$and $\mathrm{A}^{+}$from laser induced fluorescence matrix isolation spectroscopy, but their values ( 95 and $17 \mathrm{~cm}^{-1}$, respectively) seem to be out of range (see Table IV).

\section{Vertical and adiabatic ionization energies}

Vertical and adiabatic ionization energies $\left(I E_{\mathrm{v}}\right.$ and $I E_{\mathrm{a}}$, respectively) were determined at the ic-MRCI+Q level of theory for all the cationic states of interest, using the hierarchical $\mathrm{V} n \mathrm{Z}$ basis set series (with $n=\mathrm{Q}, 5$, and 6 ), and the individual energies were extrapolated to the CBS limit (see section II A). The $\mathrm{V} n \mathrm{Z}$ energies of all states were calculated at their corresponding AVTZ equilibrium geometries (see Table II), for evaluating the adiabatic energies. Those of the cationic states were also calculated at the AVTZ equilibrium geometry of the $\mathrm{X}$ state of $\mathrm{HC}_{5} \mathrm{~N}$, for determining the vertical energies.

The results are reported in Table $\mathrm{V}$, which shows the convergence of the ionization energies toward the CBS limit. The CBS+ZPE values refer to CBS values of $I E_{\text {a }}$ corrected for the zero point energy (ZPE) difference between the considered cationic state and the neutral ground state. We refer to Table III for the cationic ZPE values and to the value of $8360 \mathrm{~cm}^{-1}$, calculated at the same level of theory for the neutral state.

The adiabatic values are compared to our experimental TPES values and to the photoelectron spectroscopy values of Bieri et al. ${ }^{34}$ There are no theoretical values of ionization energies to our knowledge in the literature. The deviations with respect to the TPES values for the four low-lying states are given in parenthesis, showing that the CBS extrapolation 


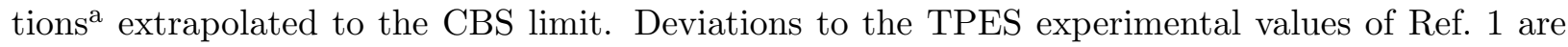
given in parentheses.

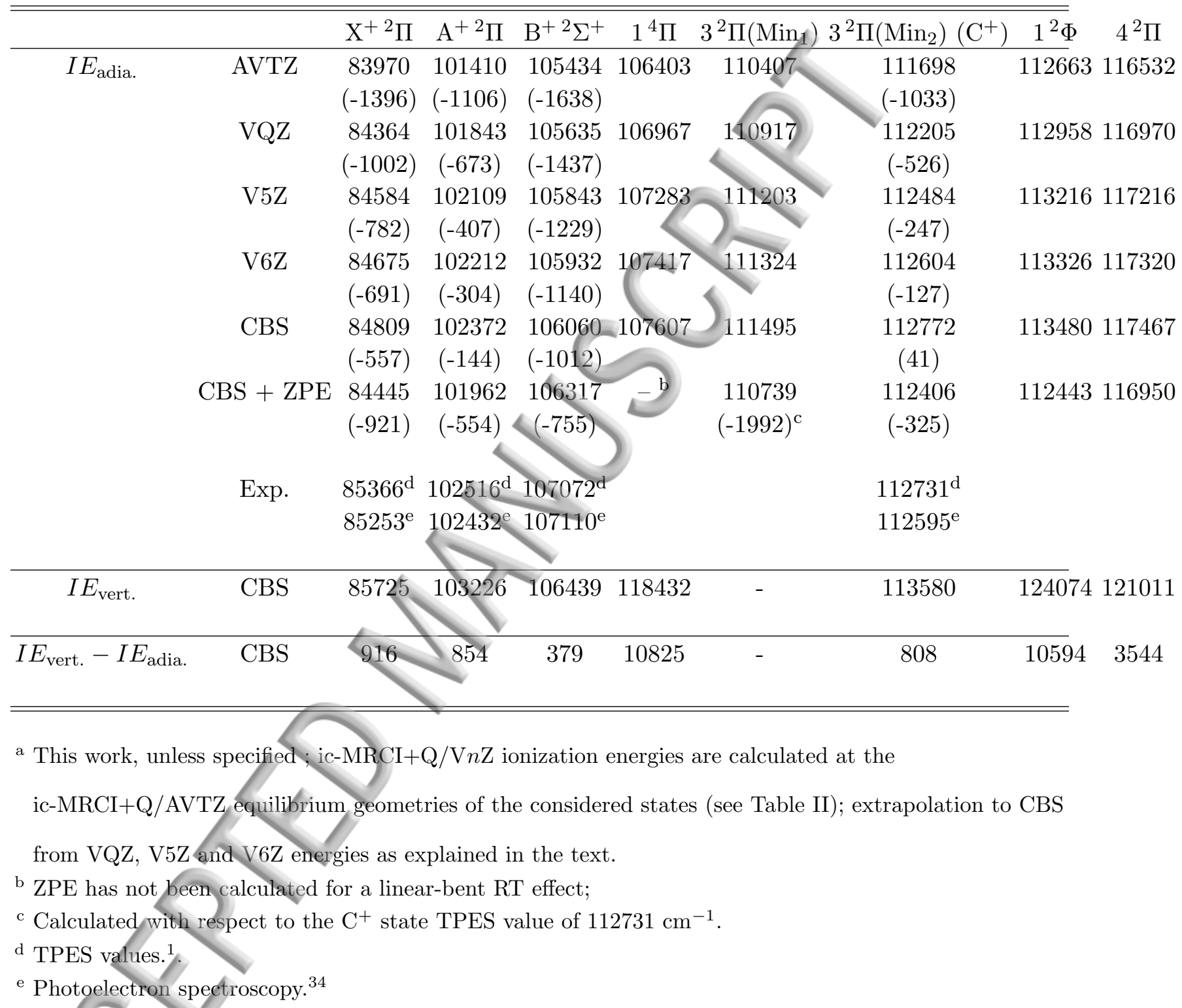

improves the convergence to the experimental values (within $\simeq 300-900 \mathrm{~cm}^{-1}$ at the CBS + ZPE level). Deviations are negative, which means that calculated $I E$ values are underestimated. This comes from the fact that the energy of the neutral ground state converges more slowly than the one of the cationic states, which involve one fewer electron. Such energy differences that imply the correlation energy balance between two systems that differ in the number of electrons are demanding, and the agreement can be considered as very 
Publishipmisfactory. Let us also note that the ZPE correction is a negative energy contribution, except in the case of the $\mathrm{B}^{+}$state for which the ZPE value is larger than that for the $\mathrm{X}$ neutral state. The CBS value for this state deviates more from TPES than the other states, but this is compensated by the positive ZPE correction, leading to a CBS + ZPE estimate lying within our ab initio error bar. For the $\mathrm{C}^{+}$state, we have here the opportunity to confirm that the observed vibrational structures arise from the $3^{2} \Pi\left(\mathrm{Min}_{2}\right)$ and not from the $3^{2} \Pi\left(\operatorname{Min}_{1}\right)$ potential well, owing to the deviations from the TPES values of 325 and $1993 \mathrm{~cm}^{-1}$, respectively.

The difference between vertical and adiabatic ionization is also given in the last row for the CBS values only. This difference is small (800-900 $\left.\mathrm{cm}^{-1}\right)$ and smaller than the ZPE values for the states identified in our TPE spectrum $\left(\mathrm{X}^{+}, \mathrm{A}^{+}, \mathrm{B}^{+}\right.$, and $\left.\mathrm{C}^{+}\right)$, see Table III. The difference is however considerably larger $\left(>10000 \mathrm{~cm}^{-1}\right)$ for the states arising from configuration (5). This is consistent with the important geometry differences pointed out before (see section III B 2), which explains why the energies reached on the PESs of the cation vertically from the $\mathrm{X}$ state are far from the bottom of the corresponding potential wells. The difference is however smaller $\left(3500 \mathrm{~cm}^{-1}\right)$ for the $4^{2} \Pi$ state, which also belongs to configuration (5), because of avoided crossings (ACs) as detailed in Section III E 2. Although the $4^{2} \Pi$ and $1^{2} \Phi$ states have similar geometries, these ACs also imply the inversion of the ionization energy order of $4^{2} \Pi$ and $1^{2} \Phi$ when going from adiabatic to vertical, which means that a state crossing must occur at intermediate geometries.

\section{Electronic transitions in $\mathrm{HC}_{5} \mathrm{~N}^{+}$cation}

\section{Transition energies}

Vertical and adiabatic transition energies $\left(T E_{\mathrm{v}}\right.$ and $T E_{\mathrm{a}}$, respectively) to the excited states of the cation were calculated using the same CBS extrapolation procedure as for the ionization energies. $T E_{\mathrm{a}}$ values are directly obtained by simple differences of the adiabatic ionization energies of Table $\mathrm{V}$, while $T E_{\mathrm{v}}$ values result from energy differences between excited and ground state cationic energies, calculated at the equilibrium geometry of $\mathrm{X}^{+}$.

The results are reported in Table VI.

The convergence toward CBS being quite similar for all states, it is illustrated for the 
\$13LE VI. Vertical $\left(T E_{\mathrm{v}}\right)$ and adiabatic $\left(T E_{\mathrm{a}}\right)$ transition energies (in $\mathrm{cm}^{-1}$ ), from ic$\mathrm{MRCI}+\mathrm{Q} / \mathrm{V} n \mathrm{Z}$ calculations extrapolated to the CBS limit.

\begin{tabular}{|c|c|c|c|}
\hline & Method $^{\mathrm{a}}$ & $T E_{\mathrm{v}}$ & $T E_{\mathrm{a}}$ \\
\hline $\mathrm{X}^{+2} \mathrm{\Pi}$ & & 0 & 0 \\
\hline \multirow[t]{8}{*}{$\mathrm{A}^{+2} \Pi$} & AVTZ & 18726 & 17440 \\
\hline & VQZ & 18681 & 17479 \\
\hline & $\mathrm{V} 5 \mathrm{Z}$ & 18702 & 17526 \\
\hline & V6Z & 18715 & 17537 \\
\hline & CBS & 18728 & 17563 \\
\hline & $\mathrm{CBS}+\mathrm{ZPE}^{\mathrm{b}}$ & & 17517 \\
\hline & Theory & $\mathrm{c}$ & \\
\hline & Exp. & & $17150^{\mathrm{e}}, 17179.5^{\mathrm{f}}, 17077.5^{\mathrm{g}}$ \\
\hline \multirow[t]{5}{*}{$\mathrm{B}^{+2} \Sigma^{+}$} & AVTZ & 21871 & 21464 \\
\hline & CBS & 21844 & 21251 \\
\hline & $\mathrm{CBS}+\mathrm{ZPE}^{\mathrm{b}}$ & & 21872 \\
\hline & Theory & & \\
\hline & Exp. & & $21706^{\mathrm{e}}$ \\
\hline \multirow[t]{2}{*}{$1^{4} \Pi$} & AVTZ & & 22433 \\
\hline & $\mathrm{CBS}$ & 227 & 22798 \\
\hline \multirow[t]{3}{*}{$3^{2} \Pi\left(\operatorname{Min}_{1}\right)$} & AVTZ & & 26437 \\
\hline & $\mathrm{CBS}$ & - & 26686 \\
\hline & $\mathrm{CBS}+\mathrm{ZPE}^{\mathrm{b}}$ & & 26294 \\
\hline \multirow{5}{*}{$\begin{array}{c}3^{2} \Pi\left(\operatorname{Min}_{2}\right) \\
\left(\mathrm{C}^{+}\right)\end{array}$} & AVTZ & 29178 & 27728 \\
\hline & CBS & 29232 & 27963 \\
\hline & 17 & & 27961 \\
\hline & Theory & $28794^{\mathrm{c}}$ & \\
\hline & Exp. & & $27365^{\mathrm{e}}$ \\
\hline \multirow[t]{4}{*}{$1^{2} \Phi$} & & 33852 & 28693 \\
\hline & & 33692 & 28671 \\
\hline & & & 27998 \\
\hline & & $32746^{\mathrm{c}}$ & \\
\hline \multirow[t]{4}{*}{$4^{2} \Pi$} & & 30767 & 32562 \\
\hline & CBS & 30853 & 32658 \\
\hline & $\mathrm{CBS}+\mathrm{ZPE}^{\mathrm{b}}$ & & 32505 \\
\hline & Theory & $28955^{\mathrm{c}}$ & \\
\hline
\end{tabular}

a This work, unless specified ; transition energies are obtained from ic-MRCI+Q/VnZ calculations performed at the ic-MRCI+Q/AVTZ equilibrium geometries of the considered states (see Table II); extrapolation to CBS has been carried out as explained in the text using the VQZ, V5Z and V6Z energies;

${ }^{\mathrm{b}}$ CBS values corrected for the ZPE contributions estimated from the ic-MRCI+Q/AVTZ harmonic frequencies calculated in this work;

${ }^{c}$ From Ref. 5; MRCI+Q/DZVP calculations performed at the B3LYP/6-311G* equilibrium geometry of the ground state;

${ }^{\mathrm{d}}$ From Ref. 4; CASPT2/DZVP calculations performed at the UHF/4-31G equilibrium geometry of the ground state;

${ }^{\text {e }}$ Calculated as the difference of TPES adiabatic ionization energies (this work, Table 1 of Paper $\mathrm{I}^{1}$ ) 
Publishi.Ag state only. One sees that the energy improvement from AVTZ to CBS is smaller for TE values $\left(<400 \mathrm{~cm}^{-1}\right)$, as compared with $I E$ values $\left(<1200 \mathrm{~cm}^{-1}\right)$, see Tables V and VI. As already pointed out, $I E$ values result from energy differences between systems having a different number of correlated electrons and are thus more demanding to compute than $T E$ values which correspond to energy differences within the same system. Unfortunately, the extrapolation increases slightly the deviation with respect to the experimental $T E$ values in the case of the $\mathrm{A}^{+}-\mathrm{X}^{+}$and $\mathrm{C}^{+}-\mathrm{X}^{+}$transitions. This comes from the more important basis set improvement of the ground state energy.

As in Table $\mathrm{V}, \mathrm{CBS}+\mathrm{ZPE}$ values refer to $T E_{\mathrm{a}}(\mathrm{CBS})$ values corrected by the ZPE of Table III.

Comparison with direct spectroscopic values ${ }^{32,33,35}$ is limited to the $\mathrm{A}^{+}$state, with deviation of less than $450 \mathrm{~cm}^{-1}$. Indirect estimates can also be obtained by differences of our TPES $I E_{\mathrm{a}}$ values. Comparison with other theoretical work is limited to $T E_{\mathrm{v}}$ values obtained at a lower level of theory. ${ }^{4,5}$ The agreement is reasonable (within $2000 \mathrm{~cm}^{-1}$ ) and the energy order is the same. However, let us recall the energy inversion between $1^{2} \Phi$ and $4^{2} \Pi$ states from vertical to adiabatic transition.

\section{Electronic transition dipole moments}

Electronic transition electric dipole moments between the four lowest electronic states of the cation are reported in Table VII. These values have been calculated at the icMRCI/AVTZ/level at the equilibrium geometries of the considered states. The geometry dependency is found to be small, which demonstrates the validity of the Franck-Condon approximation. The intensity of an electronic transition being proportional to the square of the corresponding transition moment value, one can predict the relative intensities of the different electronic transitions which may be observed in future absorption or emission spectroscopy experiments. The $\mathrm{X}^{+} \leftrightarrow \mathrm{A}^{+}$transition is clearly the most intense, followed by the $\mathrm{A}^{+} \leftrightarrow \mathrm{C}^{+}$and $\mathrm{X}^{+} \leftrightarrow \mathrm{C}^{+}$transitions, about 40 and $80 \%$ weaker, respectively. Probabilities

of transitions between $\mathrm{B}^{+2} \Sigma^{+}$and any of the ${ }^{2} \Pi$ states are more than $95 \%$ weaker and are suspected to be unobservable. 
BLE VII. Square of the transition dipole moments (in Debye ${ }^{2}$ ), from ic-MRCI/AVTZ calculations performed at the equilibrium geometries of the different $\mathrm{HC}_{5} \mathrm{~N}^{+}$electronic states.

\begin{tabular}{lcccc}
\hline \hline & \multicolumn{3}{c}{ geometry } & \\
\cline { 2 - 5 } & $\mathrm{X}^{+}$ & $\mathrm{A}^{+}$ & $\mathrm{B}^{+}$ & $\mathrm{C}^{+}$ \\
\hline$<\mathrm{X}^{+}|\mu| \mathrm{A}^{+}>^{2}$ & 17.72 & 22.77 & 20.70 & 22.69 \\
$<\mathrm{X}^{+}|\mu| \mathrm{B}^{+}>^{2}$ & 0.10 & 0.10 & 0.10 & 0.09 \\
$<\mathrm{X}^{+}|\mu| \mathrm{C}^{+}>^{2}$ & 4.08 & 3.81 & 3.14 & 3.68 \\
$<\mathrm{A}^{+}|\mu| \mathrm{B}^{+}>^{2}$ & 0.09 & 0.08 & 0.09 & 0.08 \\
$<\mathrm{A}^{+}|\mu| \mathrm{C}^{+}>^{2}$ & 12.15 & 13.30 & 13.22 & 16.31 \\
$<\mathrm{B}^{+}|\mu| \mathrm{C}^{+}>^{2}$ & 0.04 & 0.03 & 0.04 & 0.04 \\
\hline \hline
\end{tabular}

\section{Franck-Condon simulations of vibronic transitions in $\mathrm{HC}_{5} \mathrm{~N}^{+}$cation}

To test and exploit the stretching vibrational frequencies calculated in the present paper, we have performed Franck-Condon Factor (FCF) simulations of the $\mathrm{A}^{+} \rightarrow \mathrm{X}^{+}$emission spectrum in order to compare with other available experimental spectra. As explained in Paper $\mathrm{I}^{1}$, only stretching vibrational modes of the $\mathrm{HC}_{5} \mathrm{~N}^{+}$cation were observed in the TPE spectrum and not all of them were seen. The same situation is encountered in the $\mathrm{A}^{+} \leftrightarrow \mathrm{X}^{+}$ system of the cation ${ }^{33,34}$ Bieri et al. $^{34}$ recorded the gas-phase emission spectrum of $\mathrm{HC}_{5} \mathrm{~N}^{+}$ formed electronically excited in the $\mathrm{A}^{+}$state from neutral cyanobutadiyne by interaction with an electron beam. The explored range spanned roughly $2500 \mathrm{~cm}^{-1}$ below the vibrationless state of $\mathrm{A}^{+}$. Smith et al. ${ }^{33}$ investigated the $\mathrm{A}^{+} \rightarrow \mathrm{X}^{+}$transition by Laser-induced Fluorescence (LIF) spectroscopy in neon matrix environment in a range completing the data of Bieri et al. ${ }^{34}$ Figure 3 shows the results for the emission spectrum of Bieri et al. ${ }^{34}$ The calculated spectrum, taking into account the FCF simulations and the instrumental resolution ( $F W H M$ of $50 \mathrm{~cm}^{-1}$ ), reproduces remarkably well the experimental spectrum. It confirms the assignment for the $\nu_{6}, \nu_{5}$ and $2 \nu_{6}$ bands. It enables proposal of an alternative assignment for the $\nu_{3}$ band which had been attributed to the $\nu_{4}$ band by Bieri et al. ${ }^{34}$ In addition, a very weak combination band $\nu_{5}+\nu_{6}$ is identified and proposed.

Figure 4 displays equivalent results for the LIF spectrum of Smith et $a l .{ }^{33}$ with a better spectral resolution $\left(20 \mathrm{~cm}^{-1}\right)$ provided by the laser source. The agreement between the sim- 
Publishimgted spectrum and the experimental one is quite good for the band positions. It confirms almost all of the the assignments except for the $\nu_{1}$ band and the $\nu_{2}+\nu_{4}$ combination band which are instead assigned to $\nu_{2}+\nu_{5}$ and $2 \nu_{3}$ bands, respectively. The simulations validate the quality of our ab initio results, already pointed out in Section III B 1.

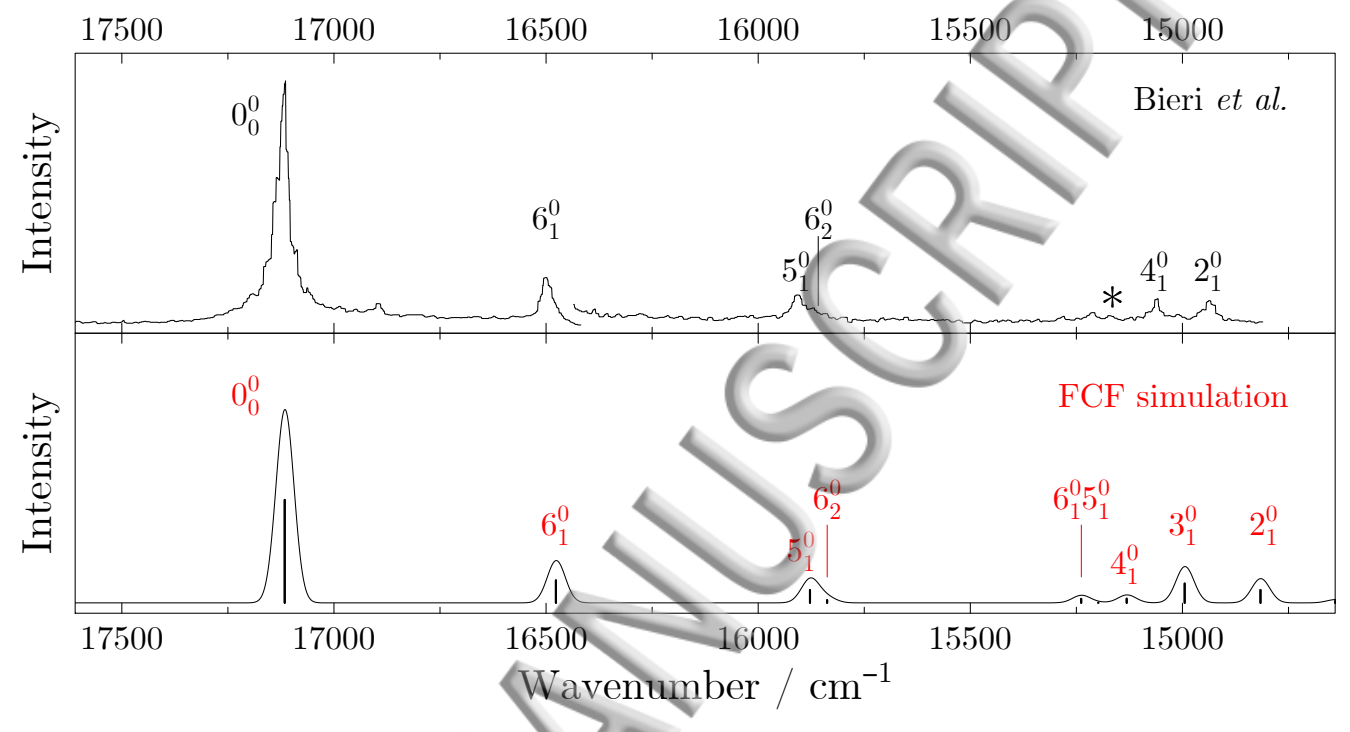

FIG. 3. Comparison of our calculated spectrum (lower panel) with the experimental $\mathrm{A}^{+} \rightarrow \mathrm{X}^{+}$ emission spectrum of Bieri et al. (upper panel). ${ }^{28}$ The black labels are reported from Ref. 28 and the star symbol corresponds to the position of the strong Lyman- $\alpha$ emission line of hydrogen which has been removed for the sake of clarity. In the lower panel, the calculated black sticks have been convoluted with a gaussian-line shape $\left(F W H M=50 \mathrm{~cm}^{-1}\right)$ to reproduce the experimental bandwidth. The red labels are deduced from our FCF calculation (see text).

\section{E. Topography of the crossing potential energy surfaces}

We are back now to the perturbed energy region $3 \mathrm{eV}$ above the ground state of the cation, where our calculations predict state mixing and potential surface crossings. This situation results from the existence of numerous interacting states with different equilibrium geometries (see Section III B 2). The potential wells of two states having similar geometries are usually well nested into each other, without surface crossings in the vicinity of the surface minima. In contrast, states with staggered minima will cross at an intermediate geometry at 


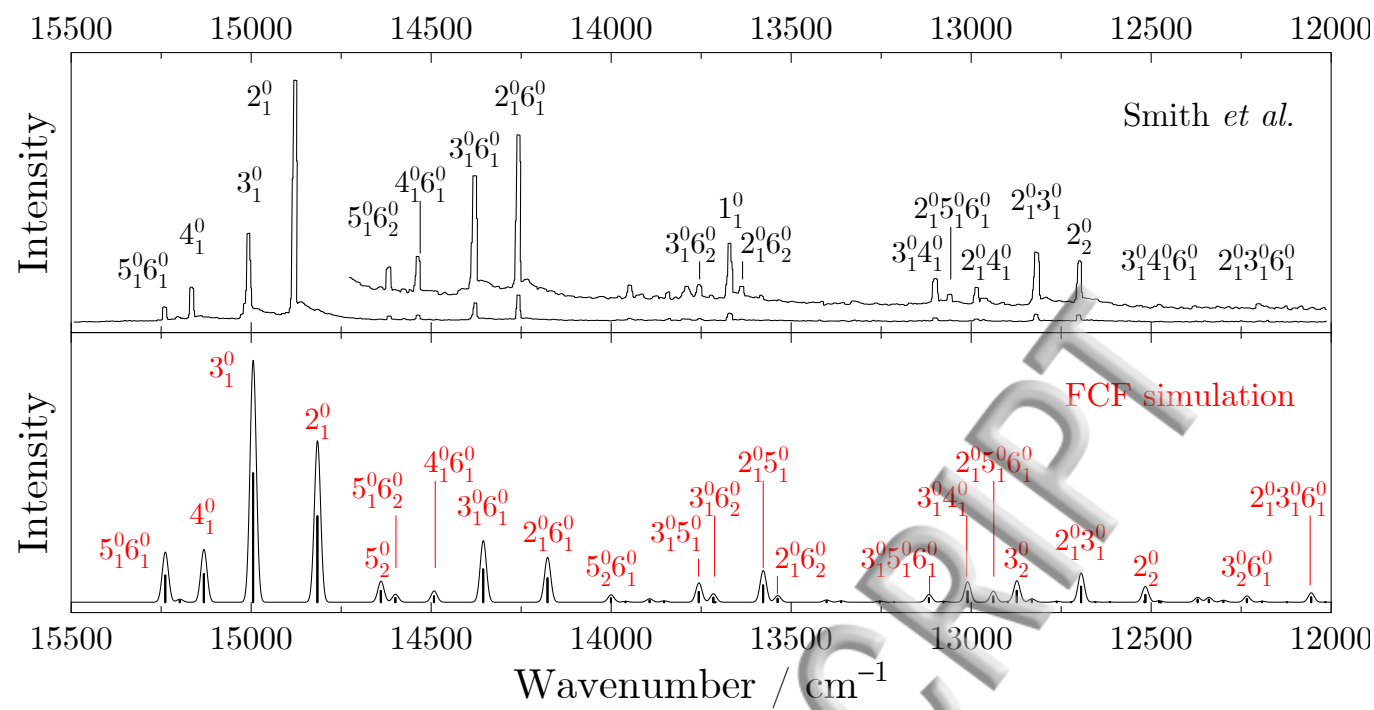

FIG. 4. Comparison of our calculated spectrum (lower panel) with the experimental $\mathrm{A}^{+} \rightarrow \mathrm{X}^{+} \mathrm{LIF}$ spectrum of Smith et al. (upper panel). ${ }^{33}$ The black labels are reported from Ref. 33 and the red ones are deduced from our FCF calculation (see text). In the lower panel, the calculated black sticks have been convoluted with a gaussian-line shape $\left(F W H M=20 \mathrm{~cm}^{-1}\right)$ to reproduce the experimental bandwidth.

energies between their respective minima. Of course, if the considered states have the same symmetry and as long as the adiabatic approximation holds, avoided crossings will occur. However, if the interaction at the crossing is symmetry-forbidden, a conical intersection may nevertheless happen, depending on symmetry conditions.

\section{Crossing pattern}

The situation that we have described is encountered here, where the PESs corresponding to the states arising from configurations (2), (3) and (4) $\left(\mathrm{A}^{+2} \Pi, \mathrm{B}^{+2} \Sigma^{+}\right.$, and $\left.3^{2} \Pi\right)$ crosses the PESs of states $\left(4^{2} \Pi, 5^{2} \Pi, 1^{2} \Phi\right.$, and $\left.1^{4} \Pi\right)$ arising from configuration (5). The geometry difference characterizing the states that belong to these two sets of configurations (see Table II) explains the observed crossings and in particular the existence of a double well in the $3^{2} \Pi$ PES. This is illustrated in Figure 5, where cuts through the PESs computed at the ic-MRCI+Q/AVTZ level are shown. The seven potential energy curves (PECs) drawn on this figure give a complete overview of the crossing pattern. The ground electronic state 


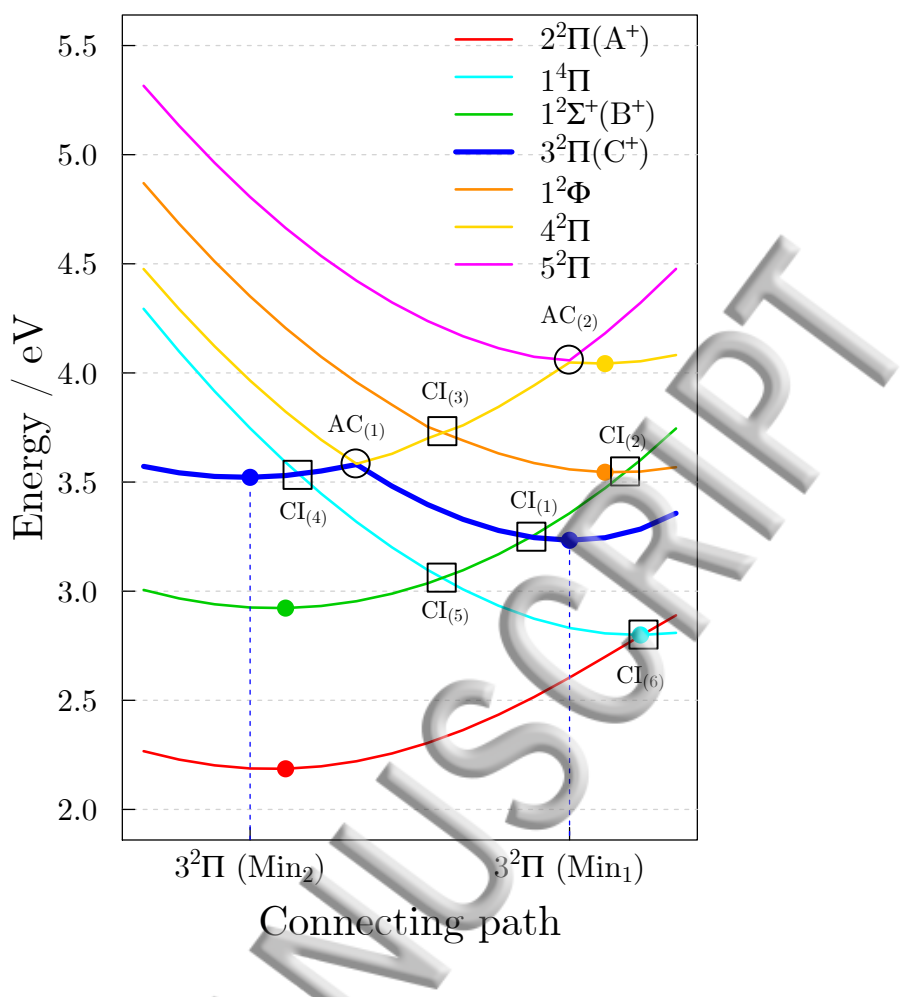

FIG. 5. Cuts through lowest excited PESs of $\mathrm{HC}_{5} \mathrm{~N}^{+}$computed at the ic-MRCI+Q / AVTZ level of theory and connecting linearly the equilibrium geometries of the $3^{2} \Pi\left(\operatorname{Min}_{1}\right)$ and the $3{ }^{2} \Pi\left(\operatorname{Min}_{2}\right)$ $\left(\mathrm{C}^{+}\right)$minima. The coloured points locate local minima along the connecting path. See text for details.

$\left(\mathrm{X}^{+2} \mathrm{\Pi}\right)$ is not represented because it lies outside of the crossing region.

The origin of the energy scale is the minimum of the $\mathrm{X}^{+}$state and the coordinate axis corresponds to a path linearly connecting the equilibrium geometries of the two minima of the $3{ }^{2} \Pi \mathrm{PES}, \mathrm{Min}_{1}$ and $\mathrm{Min}_{2}$, indicated on the abscissa axis. All bond distances were thus adjusted linearly between their equilibrium values at both minima. Sixteen points have been calculated along this path, which is representative of the crossing pattern, as all states of the two sets of crossing states have geometries which are close to those of $\operatorname{Min}_{1}$ and $\operatorname{Min}_{2}$, respectively.

The positions of the minima of the interacting ${ }^{2} \Pi$ states can be visualized on the figure. The double well $3^{2} \Pi$ PES is highlighted by a bold blue line, with each minimum indicated by a blue point. The minimum on the $4^{2} \Pi$ yellow curve is shown by a yellow circle at about $4 \mathrm{eV}$. However no minimum appears on the $5^{2} \Pi$ light blue curve, which explains 
Publishiwgy our geometry optimization did not converge, as pointed out in Section III B 1. Eight surface crossings occur between the adiabatic PESs: two of them, marked by open circles and noted as $\mathrm{AC}_{(1)}$ and $\mathrm{AC}_{(2)}$, correspond to avoided crossings $(\mathrm{AC})$ between ${ }^{2} \Pi$ states, while the others, marked by open squares and noted as $\mathrm{CI}_{(1)}$ to $\mathrm{CI}_{(6)}$, are explicit crossings between states of different spin and/or orbital symmetries, but potentially leading to conical intersections (CI) upon deformations away from linearity.

This graph generates many comments which are developped in the next subsections.

\section{Avoided crossings and diabaticity}

$\mathrm{AC}_{(1)}$ and $\mathrm{AC}_{(2)}$ arise from the adiabatic mixing of the $3{ }^{2} \Pi$ state with the $4{ }^{2} \Pi$ and 5 ${ }^{2} \Pi$ states, respectively. The evolutions of the weights of the most relevant configurations (2), (4), (5), and (6) along the reaction coordinate are illustrated in Figure 6. The most spectacular effect is the occurence of sharp jumps at points $\mathrm{AC}_{(1)}$ and $\mathrm{AC}_{(2)}$, in the (b), (c), and (d) panels. These surface hoppings are induced by configuration switches between $3^{2} \Pi$ and $4^{2} \Pi$ at $\mathrm{AC}_{(1)}$ and between $4{ }^{2} \Pi$ and $5^{2} \Pi$ at $\mathrm{AC}_{(2)}$. The complementary patterns on the (b) and (c) panels indicate a configuration exchange between (4) and (5), which cross diabatically. Configuration (2), which describes the $\mathrm{A}^{+}$state, also contributes to the electronic changes, as shown in (a), with a progressive gain of weight when approaching the $3^{2} \Pi\left(\operatorname{Min}_{1}\right)$ region.

The sharp configuration exchanges just pointed out indicate a quasi-diabatic behaviour of the involved electronic states, confirmed by the very small value of the Hamiltonian offdiagonal matrix element at $\mathrm{AC}_{(1)}$ and $\left.\mathrm{AC}_{(2)}\left(<50 \mathrm{~cm}^{-1}\right)\right)$, and explained by a bielectronic interaction involying three different orbitals $(1 \pi, 3 \pi$, and $4 \pi)$.

\section{Conical interactions}

The existence of explicit crossings $\mathrm{CI}_{(1)}$ to $\mathrm{CI}_{(6)}$ (see Figure 5) and their positions with respect to the topography of the different PESs merits special attention, because they are related to the formation of conical intersections, which may complicate the interpretation of the rovibrational spectra. Crossing point $\mathrm{CI}_{(1)}$ is of major concern in this context, as it 
Connecting path

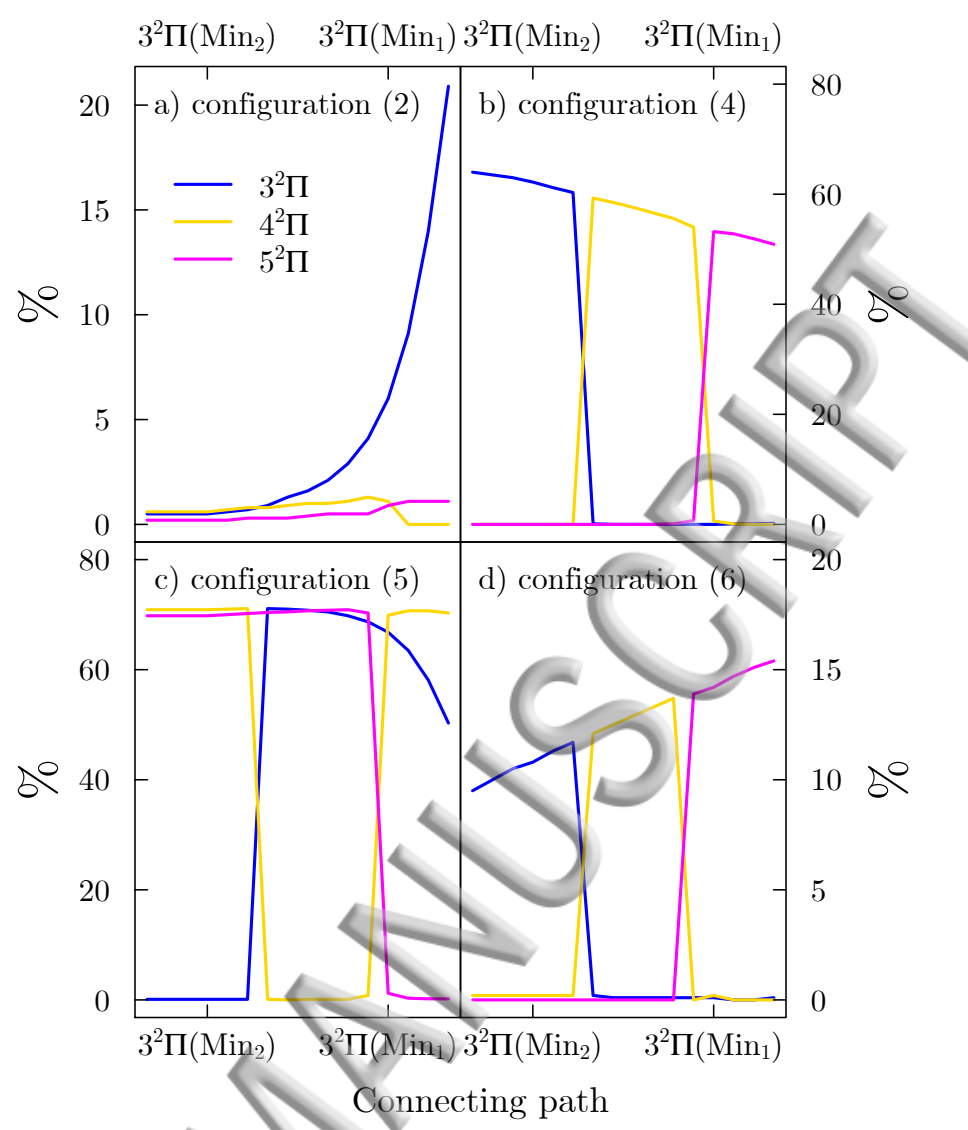

FIG. 6. Weight evolutions of the configurations (2), (4), (5), and (6) for the $3^{2} \Pi, 4^{2} \Pi$, and $5^{2} \Pi$ states of $\mathrm{HC}_{5} \mathrm{~N}^{+}$along the connecting path adopted in Figure 5.

involves the $\mathrm{B}^{+}$and $\mathrm{C}^{+}$states, which are experimentally observed. On a theoretical point of view, this is a ${ }^{2} \Sigma^{+} /{ }^{2} \Pi$ crossing, exactly like the $\mathrm{A}^{+} / \mathrm{B}^{+}$crossing in $\mathrm{HC}_{3} \mathrm{~N}^{+} .{ }^{11}$ These states obey the following $\mathrm{C}_{\infty \mathrm{v}} \rightarrow \mathrm{C}_{\mathrm{s}}$ correlation rules upon planar bending of the molecular frame: ${ }^{2} \Pi \rightarrow{ }^{2} \mathrm{~A}^{\prime} \oplus{ }^{2} \mathrm{~A}^{\prime \prime}$ and ${ }^{2} \Sigma^{+} \rightarrow{ }^{2} \mathrm{~A}^{\prime}$. The pair of ${ }^{2} \mathrm{~A}^{\prime}$ states so formed repel each other at non-linear geometries, forming a conical intersection. This is a typical case of accidental conical intersection, sometimes referred to by analogy as a pseudo-Jahn-Teller effect. ${ }^{11} \mathrm{CI}_{(1)}$ is thus the top of a double cone and belongs to the intersection seam of the CI. It is also a point of the ${ }^{2} \mathrm{~A}^{\prime \prime} \mathrm{PES}$ and there is a triple degeneracy at this geometry. The ${ }^{2} \mathrm{~A}^{\prime \prime} \mathrm{PES}$ is not perturbed by the symmetry breaking and goes through the double cone without deformations (see Fig. 8 of Ref. 11 to visualize this specific topography). It is important to note that the linear equilibrium geometries of both crossing states $\left(\mathrm{B}^{+}\right.$and $\left.\mathrm{C}^{+}\right)$are linked 
Publishingough a connecting path on the lower ${ }^{2} \mathrm{~A}^{\prime} \mathrm{PES}$.

Crossing $\mathrm{CI}_{(2)}$ between the $\mathrm{B}^{+2} \Sigma^{+}$and $1^{2} \Phi$ PESs is expected to lead to a similar result, as the correlation rule ${ }^{2} \Phi \rightarrow{ }^{2} \mathrm{~A}^{\prime} \oplus{ }^{2} \mathrm{~A}^{\prime \prime}$ is the same as in the case of a ${ }^{2} \Pi$ state. The consequence on $\mathrm{B}^{+}$is probably less important because the crossing happens at higher energy, but it may be decisive for $1^{2} \Phi$, as it occurs close to its equilibrium geometry.

Intersection point $\mathrm{CI}_{(3)}$ corresponds to a ${ }^{2} \Pi /{ }^{2} \Phi$ crossing, obeying the correlation rule ${ }^{2} \Pi /{ }^{2} \Phi \rightarrow\left({ }^{2} \mathrm{~A}^{\prime} \oplus{ }^{2} \mathrm{~A}^{\prime \prime}\right) /\left({ }^{2} \mathrm{~A}^{\prime} \oplus{ }^{2} \mathrm{~A}^{\prime \prime}\right)$, which leads to a topography even more complex, in which two conical intersections $\left({ }^{2} \mathrm{~A}^{\prime} /{ }^{2} \mathrm{~A}^{\prime}\right.$ and $\left.{ }^{2} \mathrm{~A}^{\prime \prime} /{ }^{2} \mathrm{~A}^{\prime \prime}\right)$ share the same intersection seam.

\section{Spin-orbit crossing states}

The three last crossing points $\mathrm{CI}_{(4)}$ to $\mathrm{CI}_{(6)}$ imply doublet to quartet spin change, and as far as spin-orbit interaction is ignored, the crossing is possible. However spin-orbit interaction, even if it is weak, can intermix the $(\Lambda, S)$ components of the crossing states and lead to avoided crossings between the spin-orbit states that have the same value of the $\Omega=\Lambda+\Sigma$ quantum number, where $\Sigma$ is the projection of the spin vector on the internuclear axis. Similar mixings also occur at crossing points $\mathrm{CI}_{(1)}$ to $\mathrm{CI}_{(3)}$, involving states of the same spin, as shown in our $\mathrm{HC}_{3} \mathrm{~N}^{+}$paper, ${ }^{11}$ where a theoretical analysis of the spin-orbit mixing in the vicinity of a conical intersection is presented.

\section{Vibronic perturbations in the $C^{+}$state}

The above results can now be used to explain in more detail the vibrational assignment of the $\mathrm{C}^{+}$state TPES spectrum proposed in Paper I (see Figures 3 and 4 of Ref. 1 and related discussion). This assignment was made, supported by the FCF simulation using our $a b$ initio calculations, considering that the $\mathrm{C}^{+}$signature was originated from vibrations in the $3{ }^{2} \Pi\left(\mathrm{Min}_{2}\right)$ potential well, ignoring thus the double well shape of this PES. Observation of vibrations in a single well is a reasonable zeroth-order approximation, given the quasidiabatic behaviour of the surface crossing. Vibronic perturbations may nevertheless occur, but are expected to be weak and to affect only vibrational states that have a non-zero wave- 
Publishifugiction in the localized region where the crossing occurs. A projection of the coordinates of the crossing path of Figure 5 on the normal coordinates of the stretching modes of the $3^{2} \Pi\left(\mathrm{Min}_{2}\right)$ well gives the following contributions of the different modes: $0 \% \nu_{1}+21 \% \nu_{2}$ $+18 \% \nu_{3}+9 \% \nu_{4}+1 \% \nu_{5}+50 \% \nu_{6}$. It follows that among the vibrational signatures predicted by our FCF simulation, $\nu_{6}$ is likely to be perturbed by vibronic interactions, and $\nu_{3}$ also but to a lesser extent. The intensities of the calculated stick spectrum are actually in poor agreement for these two modes with the maximum peak intensities of the TPES. The intensity of the $6_{0}^{1}$ band is underestimated by about a factor of 12 , while the intensity of the $3_{0}^{1}$ band is overestimated by a factor of about 2. We attribute these discrepancies to the vibronic perturbations induced by the surface crossing, which have not been taken into account by the single state harmonic approach. We note however the remarkable intensity predictions (within less than $10 \%$ ) made for the other states of the cation, which are vibronically unperturbed.

\section{CONCLUSIONS}

In the present paper of this series dedicated to the photoionization of the cyanobutadiyne molecule, an extensive ab initio study has been performed to understand the complex energy landscape up to $32000 \mathrm{~cm}^{-1}$ above the ionization potential. The electronic properties of the seven low-lying electronic states of the cation have been calculated at a high level of $a b$ initio theory which allows an accurate description of the multiconfigurational effects that affect the excited electronic structure. Convergence to the CBS limit has been applied to the determination of accurate ionization and transition energies. The first goal of this work was to provide a theoretical support to the interpretation of the threshold-photoelectron spectrum of $\mathrm{HC}_{5} \mathrm{~N}$, presented in the first paper of this serie (Paper I). ${ }^{1}$ In these experiments the first four electronic states of the cation $\left(\mathrm{X}^{+}, \mathrm{A}^{+}, \mathrm{B}^{+}\right.$, and $\left.\mathrm{C}^{+}\right)$are populated by single-

photon ionization processes. The assignment of the vibrational structures that appear in this spectrum have been established in Paper I thanks to the theoretical results detailed in the present paper. The second goal of this work was to provide a more complete survey of the excited electronic structure of the cation. We have thus extended our theoretical study beyond the scope of the TPES experiment, by characterizing additional high energy states, unattainable by single-photon photoelectron spectroscopy. However these states indirectly 
Publishing tribute however to the understanding of the vibronic perturbations affecting the TPE spectrum of the $\mathrm{C}^{+}$state. In addition to the properties directly related to our photoelectron experiments (ionization energies and vibrational frequencies), we have calculated additional properties which may be helpful to guide further spectroscopy experiments dedicated to the cyanobutadiyne cation. These properties, calculated for all considered states, include the rotational constants, permanent and transition electric dipole-moments, spin-orbit constants, transition energies, and Renner-Teller parameters. The accuracy of the calculations is demonstrated through the good agreement between FCF simulations and experimental spectra, both for the photoionization and emission processes. This work contributes to the global knowledge of the photoionization of cyanopolyynes and the electronic structure of the corresponding cations of astrophysical interest. ${ }^{10-12,36-39}$

\section{ACKNOWLEDGMENTS}

J.L. thanks the Fonds National de la Recherche Scientifique de Belgique (FRS-FNRS) for financial support (IISN 4.4504.10 project) and the ULB/VUB computing center and the CECI for computational support. This work received financial support from the French Agence Nationale de la Recherche (ANR) under Grant No. ANR-17-CE30-0031 (project PRIMA) and the CNRS program "Physique et Chimie du Milieu Interstellaire" (PCMI) co-funded by the Centre National d'Etudes Spatiales (CNES).

\section{REFERENCES}

${ }^{1}$ B. Gans, N. Lamarre, J. Guillemin, S. Douin, C. Alcaraz, C. Romanzin, G. Garcia, J.Liévin, and S. Boyé-Péronne, "Vibronic structure of the cyanobutadiyne cation: IVUV photoionization study of $\mathrm{HC}_{5}$ N," J. Chem. Phys. (2019).

${ }^{2}$ S. Lee, "Density Functional Theory Study of Cyano- and Dicyanopolyacetylene Cations," The Journal of Physical Chemistry 100, 13959-13962 (1996).

${ }^{3}$ J. Tatchen and C. M. Marian, "On the performance of approximate spinorbit Hamiltonians in light conjugated molecules: the fine-structure splitting of $\mathrm{HC}_{6} \mathrm{H}^{+}, \mathrm{NC}_{5} \mathrm{H}^{+}$, and $\mathrm{NC}_{4} \mathrm{~N}^{+}$," Chemical Physics Letters 313, 351-357 (1999). 
Publishing. M. Lee and L. Adamowicz, "Ab initio calculations of electronically excited states of cyano-substituted polyacetylene cations," Spectrochimica Acta Part A: Molecular and Biomolecular Spectroscopy 57, 897-906 (2001).

${ }^{5}$ Z. Cao and S. D. Peyerimhoff, "MRD-CI Characterization of Electronic Spectra of Isoelectronic Species $\mathrm{C}_{6}^{-}, \mathrm{NC}_{4} \mathrm{~N}^{+}$, and $\mathrm{CNC}_{3} \mathrm{~N}^{+}$," The Journal of Physical Chemistry A 105, 627-631 (2001).

${ }^{6}$ D. Forney, P. Freivogel, J. Fulara, and J. P. Maier, "Electronic absorption spectra of cyanosubstituted polyacetylene cations in neon matrices," The Journal of Chemical Physics 102, 1510-1514 (1995).

${ }^{7}$ H.-J. Werner, P. J. Knowles, G. Knizia, F. R. Manby, M. Schütz, P. Celani, T. Korona, R. Lindh, A. Mitrushenkov, G. Rauhut, K. R. Shamasundar, T. B. Adler, R. D. Amos, A. Bernhardsson, A. Berning, D. L. Cooper, M. J. O. Deegan, A. J. Dobbyn, F. Eckert, E. Goll, C. Hampel, A. Hesselmann, G. Hetzer, T. Hrenar, G. Jansen, C. Köppl, Y. Liu, A. W. Lloyd, R. A. Mata, A. J. May, S. J. MeNicholas, W. Meyer, M. E. Mura, A. Nicklass, D. P. O'Neill, P. Palmieri, D. Peng K. Pflüger, R. Pitzer, M. Reiher, T. Shiozaki, H. Stoll, A. J. Stone, R. Tarroni, T. Thorsteinsson, and M. Wang, "MOLPRO, version 2015.1, a package of ab initio programs," (2015).

${ }^{8}$ H. Werner, P. J. Knowles, G. Knizia, F. R. Manby, and M. Schütz, "Molpro: a generalpurpose quantum chemistry program package," Wiley Interdisciplinary Reviews: Computational Molecular Science 2, 242-253 (2012).

${ }^{9}$ L. A. V. Mendes, S. Boyé-Péronne, U. Jacovella, J. Liévin, and D. Gauyacq, "Rydberg states of cyanoacetylene investigated by (3+1) REMPI spectroscopy in the 77,000 90,000 $\mathrm{cm}^{-1}$ energy range," Molecular Physics 110, 2829-2842 (2012).

${ }^{10}$ A. Destier, C. Romanzin, N. Lamarre, C. Alcaraz, B. Gans, D. Gauyacq, J. Liévin, and S. Boyé-Péronne, "Experimental and ab initio characterization of $\mathrm{HC}_{3} \mathrm{~N}^{+}$vibronic structure. I. Synchrotron-based threshold photo-electron spectroscopy," The Journal of Chemical Physics 145, 234310 (2016).

${ }^{11}$ B. Gans, N. Lamarre, M. Broquier, J. Liévin, and S. Boyé-Péronne, "Experimental and ab initio characterization of $\mathrm{HC}_{3} \mathrm{~N}^{+}$vibronic structure. II. High-resolution VUV PFI-ZEKE spectroscopy," The Journal of Chemical Physics 145, 234309 (2016).

${ }^{12}$ N. Lamarre, B. Gans, C. Alcaraz, B. C. de Miranda, J.-C. Guillemin, M. Broquier, J. Liévin, and S. Boyé-Péronne, "Vibronic structure of the ${ }^{2} \Pi_{u}$ ground electronic state of 
Publishingicyanoacetylene cation revisited by PFI-ZEKE photoelectron spectroscopy and ab initio calculations," Molecular Physics 113, 3946-3954 (2015).

${ }^{13} \mathrm{H}$. Werner and P. J. Knowles, "An efficient internally contracted multiconfigurationreference configuration interaction method," The Journal of Chemical Physics 89, 5803-5814 (1988).

${ }^{14}$ P. J. Knowles and H.-J. Werner, "An Efficient Method for the Evaluation of Coupling Coefficients in Configuration Interaction Calculations," Chem. Phys. Letters 145, 514$522(1988)$.

${ }^{15}$ H.-J. Werner and P. J. Knowles, "A Second Order MCSCF Method with Optimum Convergence," J. Chem. Phys. 82, 5053 (1985).

${ }^{16}$ P. J. Knowles and H.-J. Werner, "An Efficient Second Order MCSCF Method for Long Configuration Expansions," Chem. Phys. Letters 115, 259-267 (1985).

${ }^{17}$ K. R. Shamasundar, G. Knizia, and H.-J. Werner, "A new internally contracted multireference configuration interaction method," The Journal of Chemical Physics 135, 054101 (2011).

${ }^{18} \mathrm{~S}$. R. Langhoff and E. R. Davidson, "Configuration interaction calculations on the nitrogen molecule," International Journal of Quantum Chemistry 8, 61-72 (1974).

${ }^{19}$ H.-J. Werner, M. Kállay, and J. Gauss, "The barrier height of the $\mathrm{F}+\mathrm{H}_{2}$ reaction revisited: Coupled-cluster and multireference configuration-interaction benchmark calculations," The Journal of Chemical Physics 128, 034305 (2008).

${ }^{20}$ T. H. Dunning Jr., "Gaussian basis sets for use in correlated molecular calculations.I. The atoms boron through neon and hydrogen," J. Chem. Phys. 90, 1007-1023 (1989).

${ }^{21}$ R. A. Kendall, T. H. Dunning, and R. J. Harrison, "Electron affinities of the first-row atoms revisited. Systematic basis sets and wave functions," The Journal of Chemical Physies 96, 6796-6806 (1992).

${ }^{22}$ A. J. C. Varandas, "Extrapolating to the one-electron basis-set limit in electronic structure calculations," The Journal of Chemical Physics 126, 244105 (2007).

${ }^{23}$ P. Cassam-Chenaï and J. Liévin, "Alternative perturbation method for the molecular vibrationrotation problem," International Journal of Quantum Chemistry 93, 245-264 (2003).

${ }^{24}$ A. Berning, M. Schweizer, H.-J. Werner, P. J. Knowles, and P. Palmieri, "Spin-orbit matrix elements for internally contracted multireference configuration interaction wavefunctions," 
Publishing olecular Physics 98, 1823-1833 (2000).

${ }^{25} \mathrm{G}$. Schaftenaar and J. Noordik, "Molden: a pre- and post-processing program for molecular and electronic structures*," Journal of Computer-Aided Molecular Design 14, 123-134 (2000).

${ }^{26} \mathrm{C}$. M. Western, "PGOPHER: A program for simulating rotational, vibrational and electronic spectra," Journal of Quantitative Spectroscopy and Radiative Transfer 186, 221-242 (2017).

${ }^{27} \mathrm{G}$. Herzberg, Molecular spectra and molecular structure III. Electronic spectra and electronic structure of polyatomic molecules. (Van Nostrand Reinhold, 1966).

${ }^{28}$ G. Bieri, E. Kloster-Jensen, S. Kvisle, J. P. Maier, and O. Marthaler, "Emission spectra of the cations of cyanodiacetylene, methylcyanodiacetylene and ethylcyanodiacetylene in the gaseous phase. $\tilde{A}\left(\pi^{-1}\right) \rightarrow \tilde{X}\left(\pi^{-1}\right)$ band systems," J. Chem. Soc., Faraday Trans. 2 76, 676-684 (1980).

${ }^{29} \mathrm{P}$. Botschwina, A. Heyl, M. Oswald, and T. Hirano, "Ab initio anharmonic force fields and spectroscopic properties for $\mathrm{HC}_{5} \mathrm{~N}$ and $\mathrm{HC}_{5} \mathrm{NH}^{+}$, molecules of interest to astrochemistry," Spectrochimica Acta Part A: Molecular and Biomolecular Spectroscopy 53, 1079-1090 (1997).

${ }^{30}$ C. Degli Esposti, L. Bizzocchi, P. Botschwina, K. M. T. Yamada, G. Winnewisser, S. Thorwirth, and P. Förster, "Vibrationally excited states of $\mathrm{HC}_{5} \mathrm{~N}$ : millimeter-wave spectroscopy and coupled cluster/calculations," Journal of Molecular Spectroscopy 230, 185-195 (2005).

${ }^{31}$ A. J. Alexander H. W. Kroto, and D. R. M. Walton, "The microwave spectrum, substitution structure and dipole moment of cyanobutadiyne, $\mathrm{H}-\mathrm{C} \equiv \mathrm{C}-\mathrm{C} \equiv \mathrm{C}-\mathrm{C} \equiv \mathrm{N}$," Journal of Molecular Spectroscopy 62, 175-180 (1976).

${ }^{32}$ W. E. Sinclair, D. Pfluger, and J. P. Maier, "Rotationally resolved $A^{2} \Pi \leftarrow X^{2} \Pi$ electronic spectra of cyanodiacetylene and dicyanoacetylene cations," The Journal of Chemical Physics 111, 9600-9608 (1999).

${ }^{33}$ A. M. Smith, J. Agreiter, and V. E. Bondybey, "Laser-induced fluorescence of matrixisolated $\mathrm{H}-\mathrm{C} \equiv \mathrm{C}-\mathrm{C} \equiv \mathrm{C}-\mathrm{C} \equiv \mathrm{N}^{+}$and $\mathrm{H}-\mathrm{C} \equiv \mathrm{C}-\mathrm{C} \equiv \mathrm{C}-\mathrm{C} \equiv \mathrm{C}-\mathrm{C} \equiv \mathrm{N}^{+}, "$ Chemical Physics Letters 244, 379-387 (1995).

${ }^{34}$ G. Bieri and L. Asbrink, "30.4-nm He(II) photoelectron spectra of organic molecules. Part I. Hydrocarbons," Journal of Electron Spectroscopy and Related Phenomena 20, 149-167 (1980). 
Publishin'frg Bieri, E. Heilbronner, V. Hornung, E. Kloster-Jensen, J. P. Maier, F. Thommen, and

W. von Niessen, "Electronic states of substituted haloacetylene and cyanoacetylene radical cations," Chemical Physics 36, 1-14 (1979).

${ }^{36}$ S. Leach, M. Schwell, G. A. Garcia, Y. Bénilan, N. Fray, M.-C. Gazeau, F. Gaie-Levrel, N. Champion, and J.-C. Guillemin, "Ionization photophysics and spectroscopy of dicyanoacetylene," The Journal of Chemical Physics 139, 184304 (2013).

${ }^{37}$ S. Leach, G. A. Garcia, A. Mahjoub, Y. Bénilan, N. Fray, M.-C. Gazeau, F. Gaie-Levrel, N. Champion, and M. Schwell, "Ionization photophysics and spectroscopy of cyanoacetylene," The Journal of Chemical Physics 140, 174305 (2014).

${ }^{38}$ N. Lamarre, C. Falvo, C. Alcaraz, B. Cunha de Miranda, S. Douin, A. Flütsch, C. Romanzin, J.-C. Guillemin, S. Boyé-Péronne, and B. Gans, "Photoionization spectroscopy of $\mathrm{CH}_{3} \mathrm{C}_{3} \mathrm{~N}$ in the vacuum-ultraviolet range," Journal of Molecular Spectroscopy 315, 206-216 (2015).

${ }^{39}$ Z. Dai, W. Sun, J. Wang, and Y. Mo, "The Renner-Teller effect in $\operatorname{HCCCN}^{+}\left(\tilde{X}^{2} \Pi\right)$ studied by zero-kinetic energy photoelectron spectroscopy and theoretical calculations," The Journal of Chemical Physies 143, 054301 (2015). 

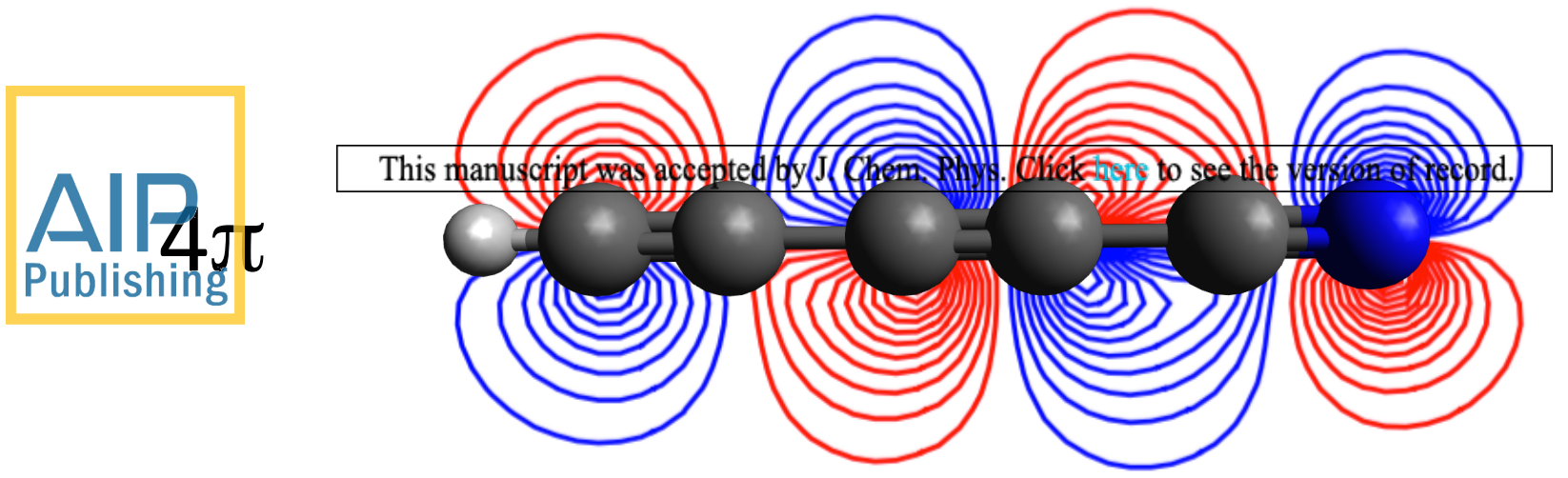

$3 \pi$
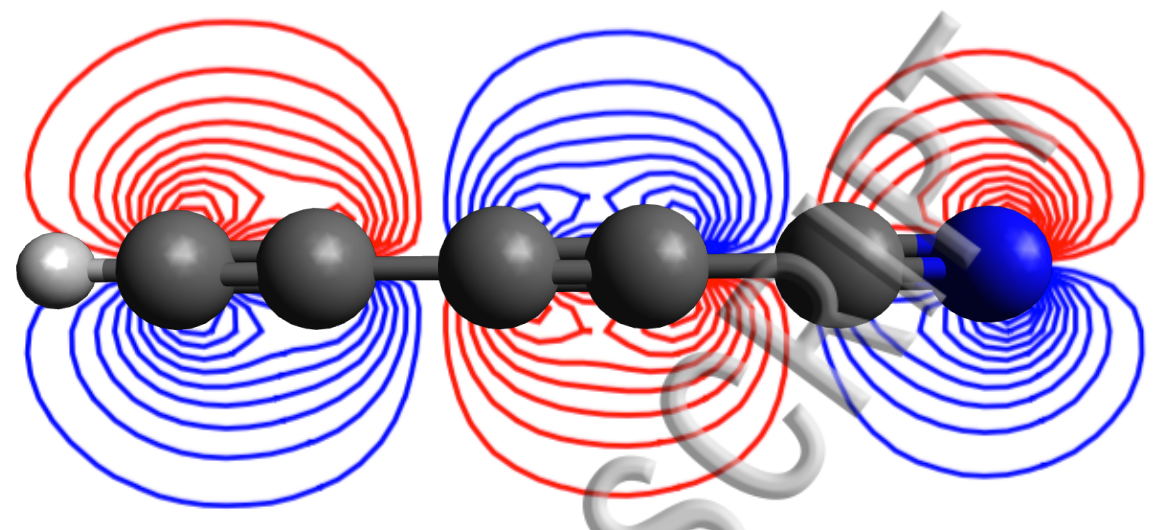

$2 \pi$
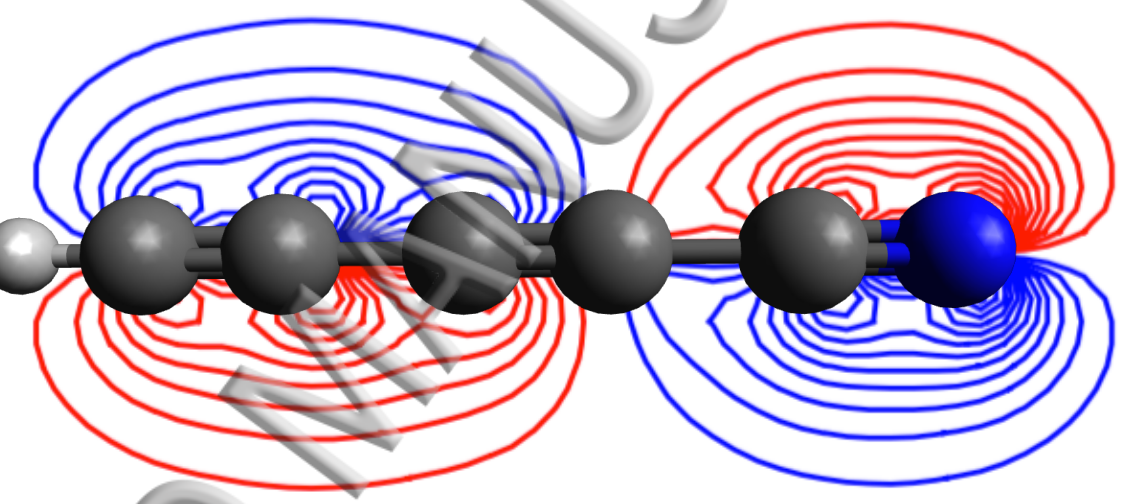

$1 \pi$
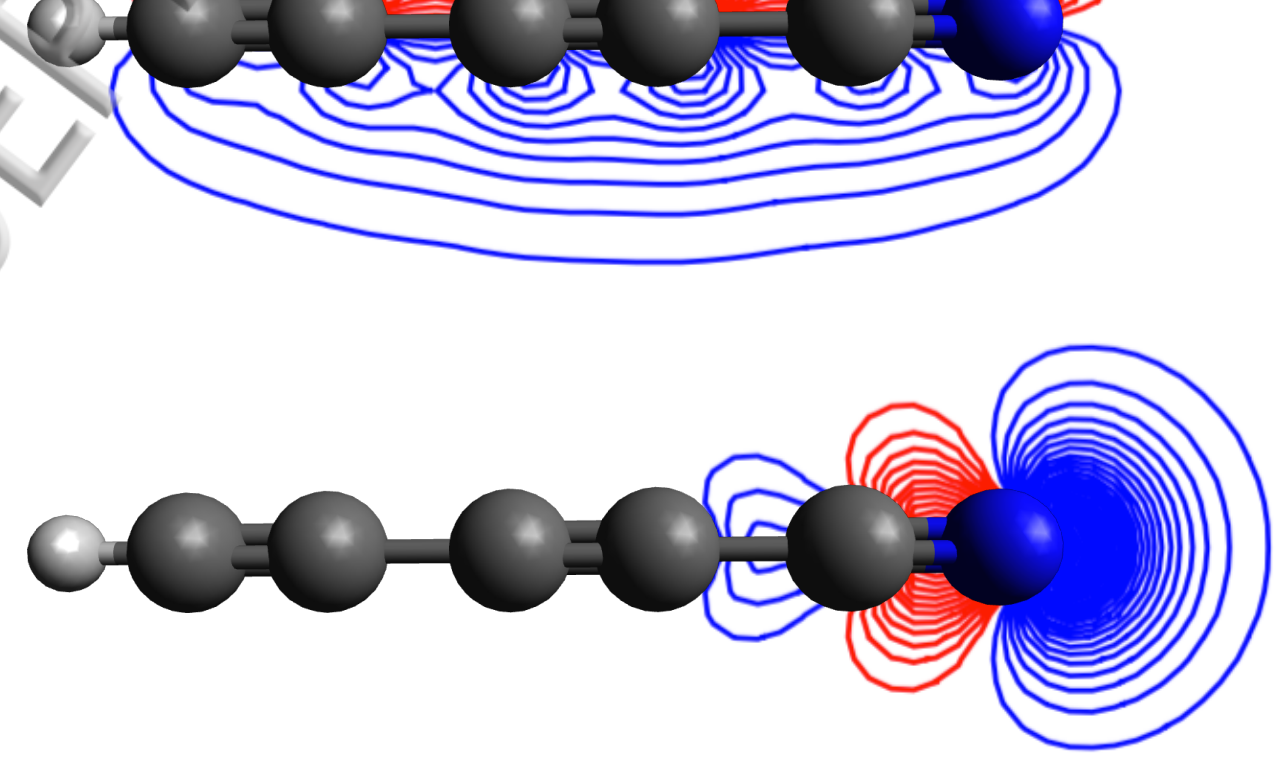


$$
X^{+2} \Pi
$$

$$
\mathrm{A}^{+2} \Pi
$$

Adpo- ब-

publishing
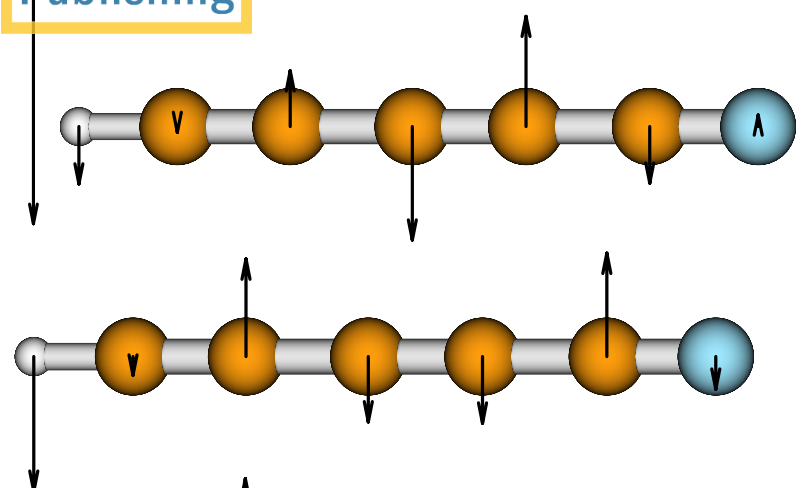

$v_{8}$
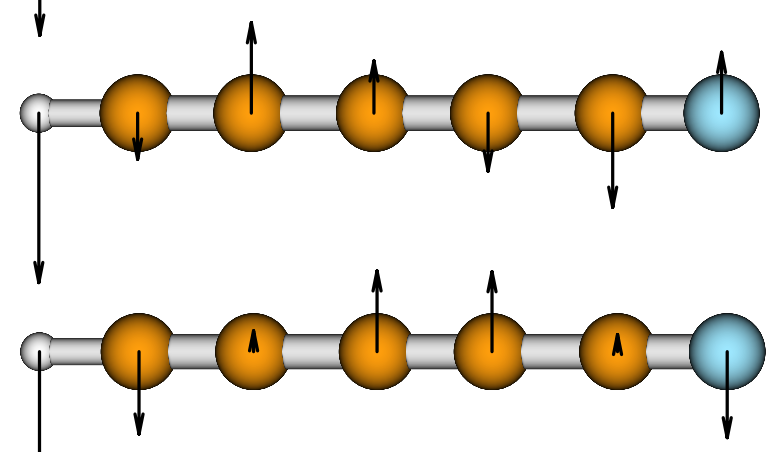

V9
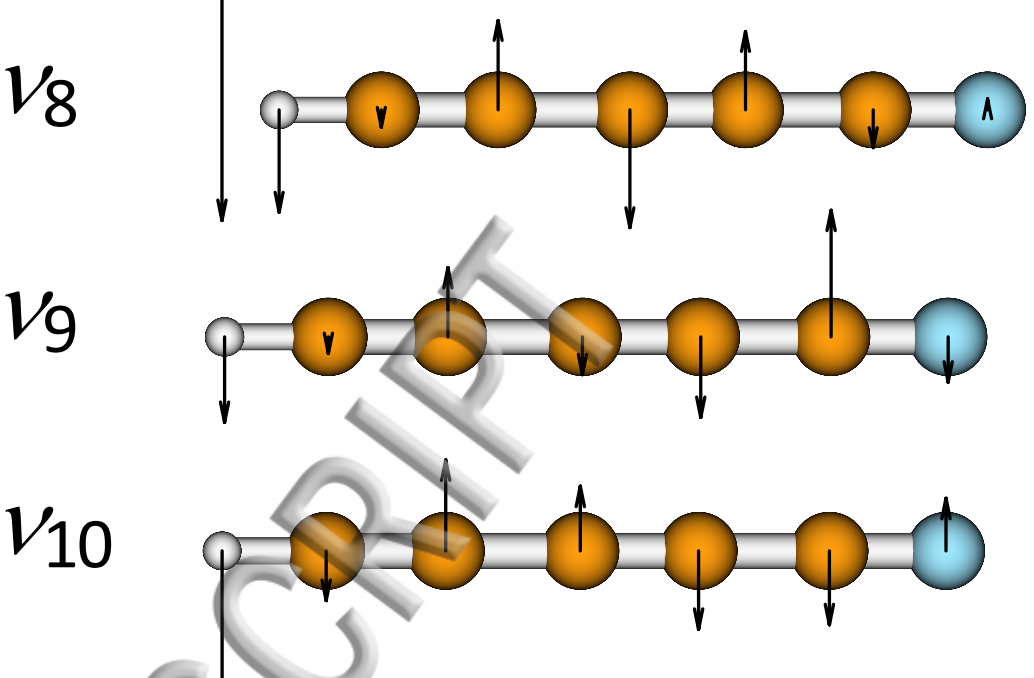

$v_{11} q Q=0-b=0-\theta$

$\mathrm{B}^{+2} \sum^{+}$

$\mathrm{C}^{+2} \Pi$

$\mathrm{F}=\mathrm{C}-\mathrm{C}-\mathrm{Cod} \mathrm{v}_{7}$
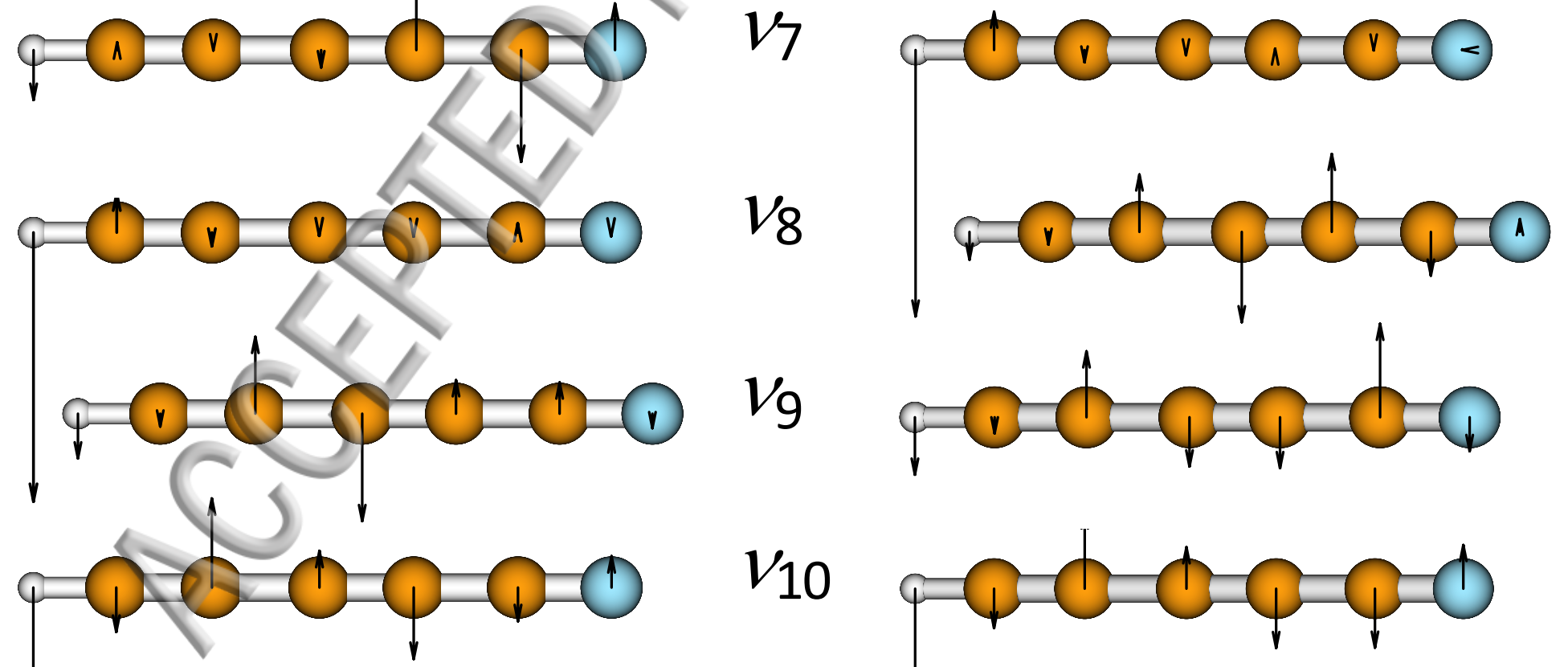

$v_{10}$
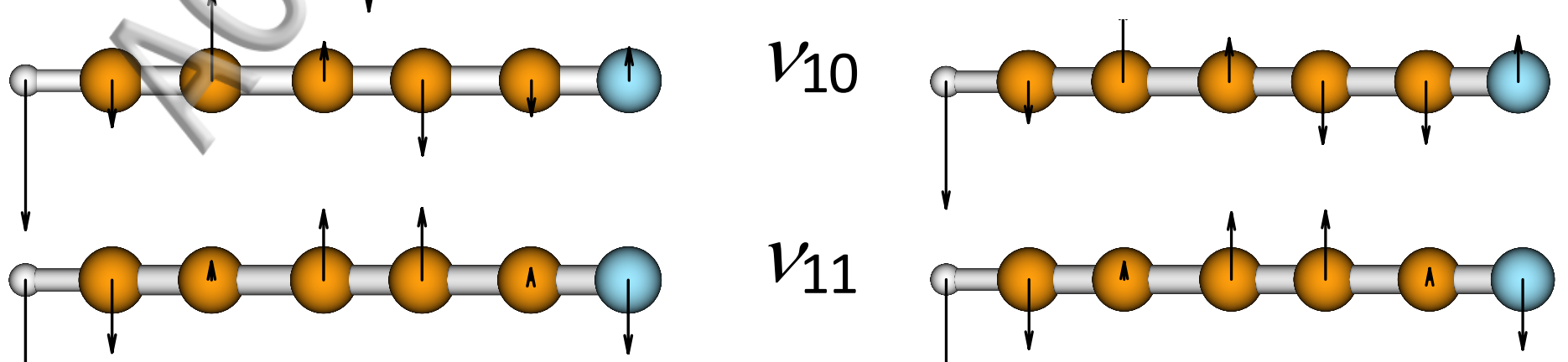

$v_{11}$

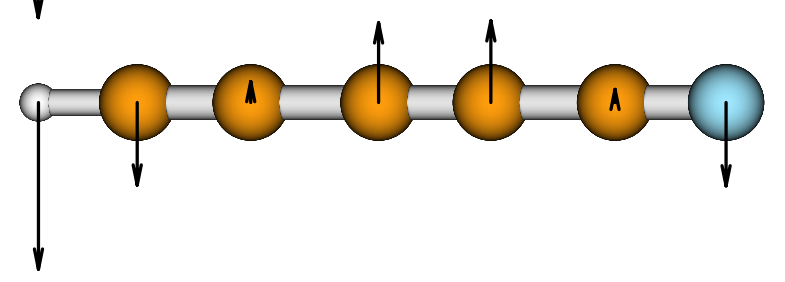




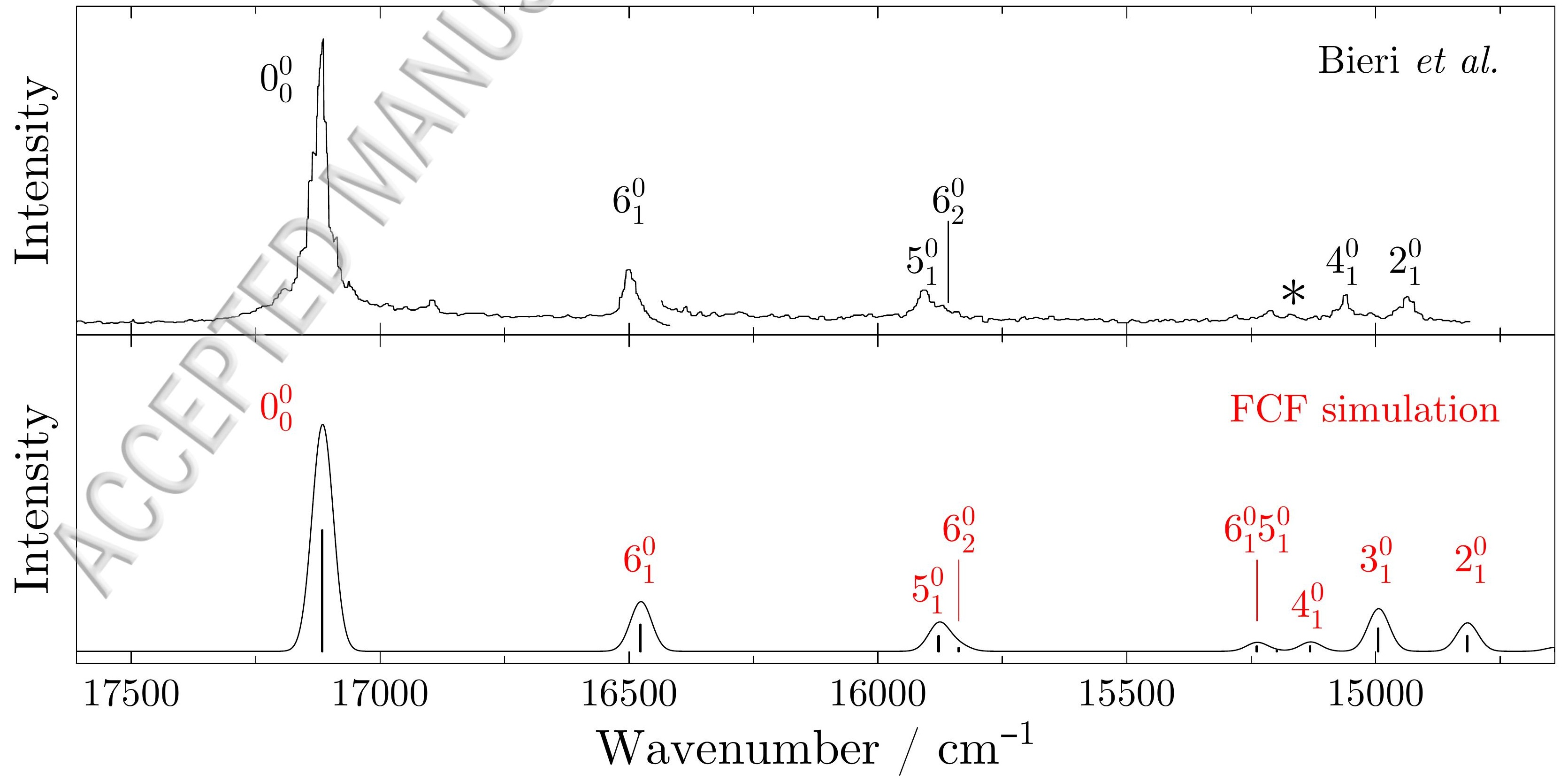




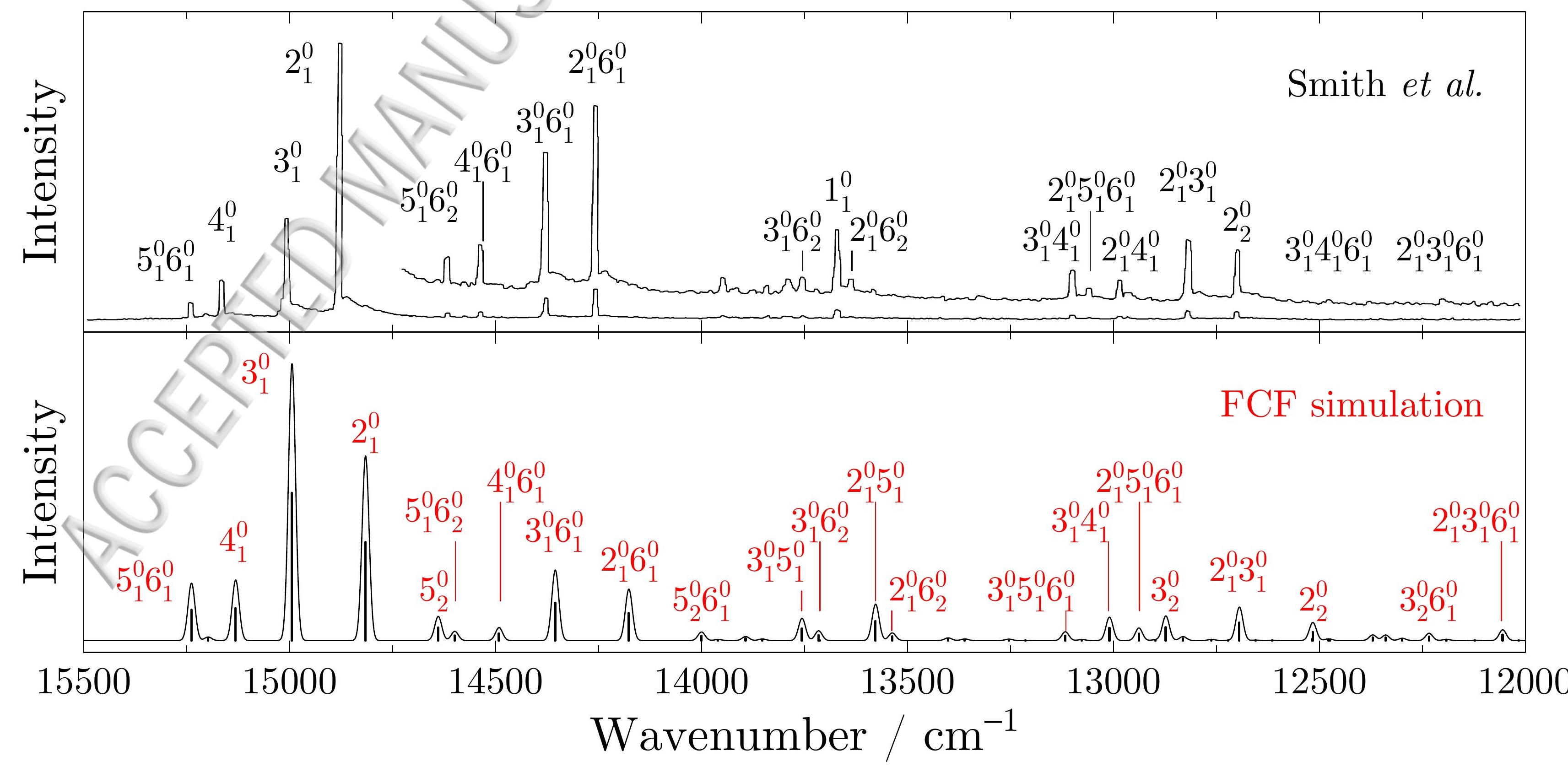




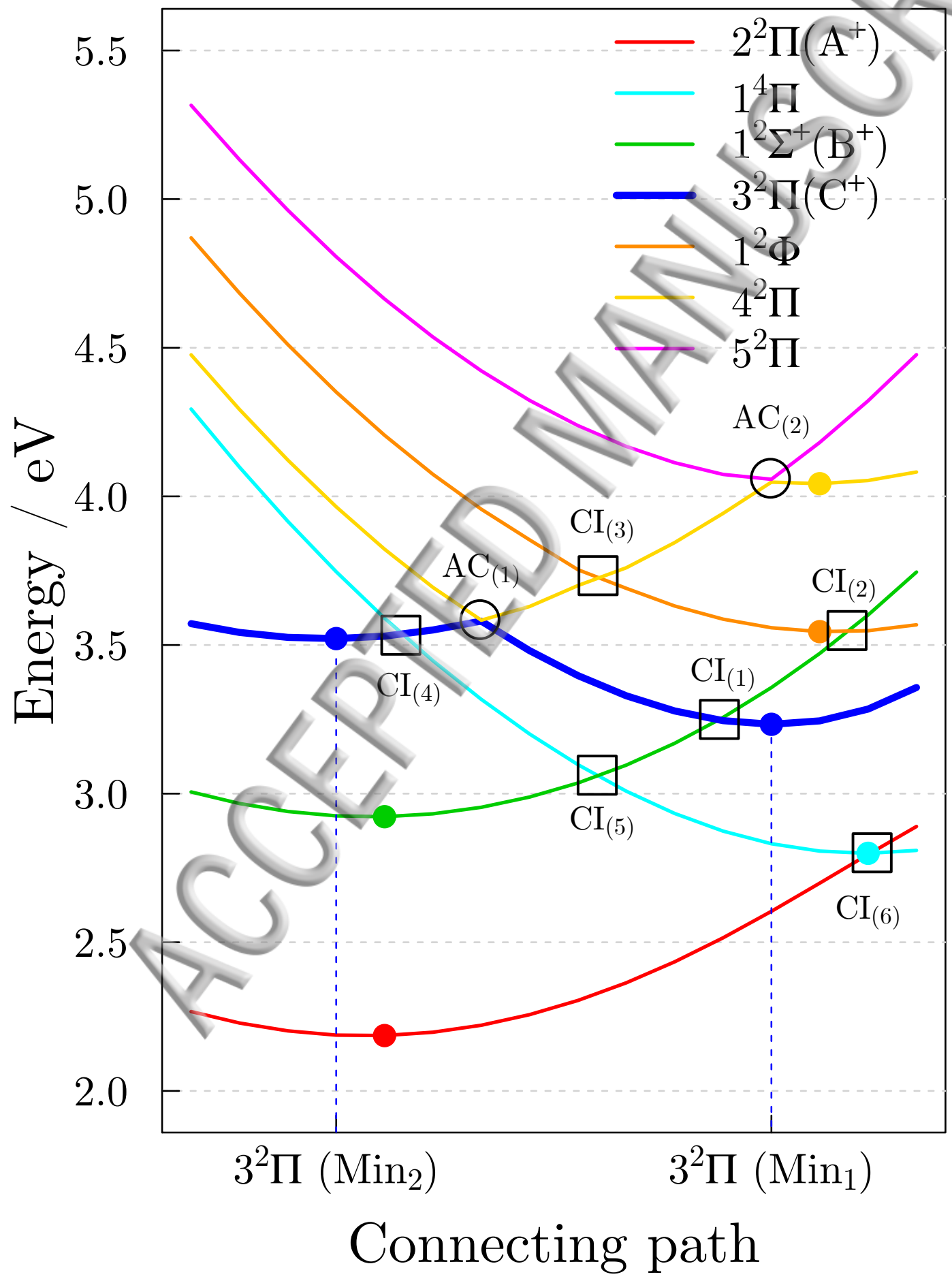


Connecting path $3^{2} \Pi\left(\operatorname{Min}_{2}\right) \quad 3^{2} \Pi\left(\operatorname{Min}_{1}\right) 3^{2} \Pi\left(\operatorname{Min}_{2}\right) \quad 3^{2} \Pi\left(\operatorname{Min}_{1}\right)$

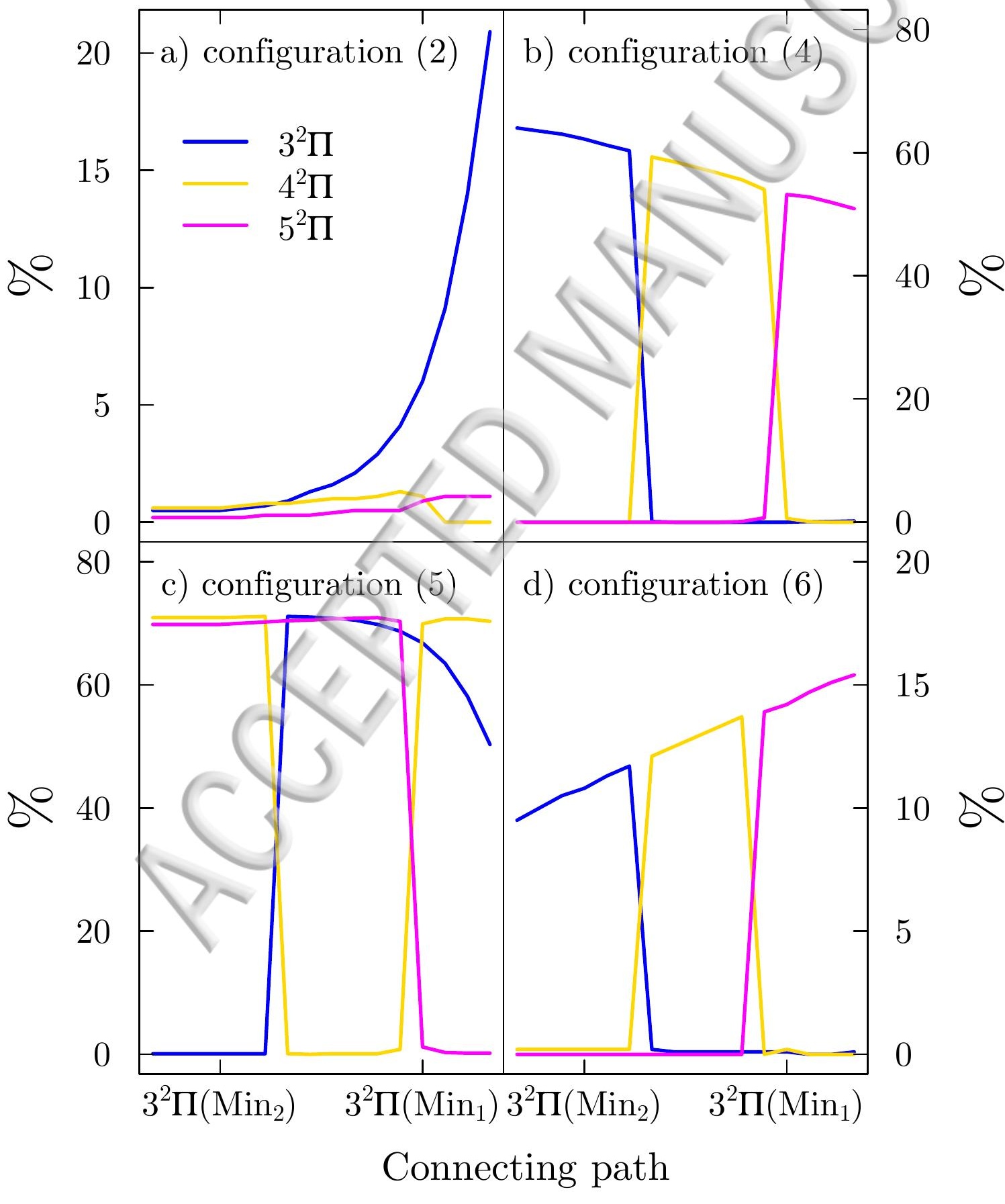

